\title{
A search for X-ray sources around WR 22 in the Carina region ${ }^{\star}$
}

\author{
J.-F. Claeskens, E. Gosset ${ }^{\star \star}$, Y. Nazé ${ }^{\star \star \star}$, G. Rauw ${ }^{\star \star \star}$, and J.-M. Vreux
}

\author{
Institute of Astrophysics and Geophysics, University of Liège, Allée du 6 août 17, 4000 Liège, Belgium \\ e-mail: gosset@astro.ulg.ac.be
}

Received 15 August 2006 / Accepted 1 June 2010

\begin{abstract}
We present the results of a deep search for X-ray sources in a circular field of $30^{\prime}$ in diameter situated around WR 22 in the Carina region and observed with the XMM-Newton observatory. This field is broadly located to the west of the main part of the Carina nebula. On the basis of six pointings (nominal exposure time: $10 \mathrm{ks}$ each) centred on the WR+O binary star WR 22, we perform a survey with an effective exposure time of $68.8 \mathrm{ks}$. We introduce a catalogue of 43 bona-fide X-ray point sources, most of which were unknown before the XMM-Newton observations, and perform the first steps towards their identifications by cross-correlating the positions with optical/infrared catalogues. We investigated the possible variability of these sources in the X-ray domain and we extracted a few X-ray spectra for the brightest ones. A short description of the diffuse X-ray emission present in the region is also given.
\end{abstract}

Key words. X-rays: general - X-rays: stars - H II regions - stars: formation

\section{Introduction}

The Carina nebula region, situated at a corner of the eponymous constellation, is certainly one of the most well-known starformation regions in the Galaxy. It is associated with a giant $\mathrm{H}$ II region that spans several square degrees and is bissected by a prominent $\mathrm{V}$-shaped dark lane: it is one of the most spectacular features in the Milky Way (see Fig. 1). Interestingly, the related Car OB1 association harbours several open clusters and/or star concentrations (Trumpler 14, 15 and 16; Collinder 228 and 232; Bochum 10 and 11) containing large numbers of massive stars and their descendants. We are dealing with a huge region of young stars, which comprises some of the hottest and presumably most massive Pop.I stars, and which features tens of massive O stars over several parsecs (see e.g. Smith 2006, and references therein). This extreme collection can be considered as a laboratory for investigating the evolution of such stars. This prominent young structure is not as compact as some of the other galactic young clusters; it provides a snapshot of an OB association in the making (Smith 2006). It is seemingly related to a spiral feature, one of the very few that are easily noticeable in the visible domain. Indeed, we are looking in this direction almost tangentially (actually a little innerly) to the now recognized Carina-Sagittarius spiral arm, which was first described in detail by Grabelsky et al. (1988). The CO survey of these authors demonstrated that the Carina nebula is at the edge of a giant molecular cloud extending over about $130 \mathrm{pc}$ and with a mass in excess of $5 \times 10^{5}$ solar masses. Large cavities within the giant molecular cloud are supposed to be carved out by Trumpler 14 and 16. This giant molecular cloud is related to the parent material (see also Brooks et al. 1998). Actually, the concentration of very massive stars interacts with the parental giant

* Based on observations with XMM-Newton, an ESA Science Mission with instruments and contributions directly funded by ESA Member States and the USA (NASA).

$\star \star$ Senior Research Associate F.R.S.-FNRS, Belgium.

$\star \star \star$ Research Associate F.R.S.-FNRS, Belgium. molecular cloud, leading to the various aspects of the region (see Cox 1995). The dark lanes consist of dust and of molecular gas associated with the nebular complex (Dickel 1974).

Various photometric studies have been performed on the open clusters of the region, in particular $\operatorname{Tr} 16$ and $\operatorname{Tr} 14$. Recently, Tapia et al. (2003) favoured a distance of $D M=12.14$ $(2.7 \mathrm{kpc})$ and an age between less than $1 \mathrm{Myr}$ and $6 \mathrm{Myr}$. DeGioia-Eastwood et al. (2001) suggested the existence in these clusters of pre-main sequence (pms) stars; intermediate-mass star formation has seemingly proceeded continuously over the last $10 \mathrm{Myr}$, whereas the massive stars observed are much younger. Another study (Carraro et al. 2004) confirmed the distance of about $2.5 \mathrm{kpc}$ for $\operatorname{Tr} 14$, but moved Tr 16 up to $4 \mathrm{kpc}$. This latter point is considered unlikely following the arguments presented by Smith (2006). On the basis of IR and radio data, Cox (1995) reported what may be the first evidence for on-going star formation in the Carina region. Megeath et al. (1996) identified a possible site. However, Cox suggested an apparent paucity of star-forming regions and/or of embedded IR regions. More recent results are challenging this view. Smith et al. (2000) reported the existence of several embedded IR sources where star formation might be going on. Brooks et al. (2001) identified two compact H II regions possibly linked to very young O-type stars. Rathborne et al. (2002) tentatively traced the photodissociation regions expected to be present in areas of massive star formation. They concluded that the star formation within the Carina region has certainly not been completely halted despite prevailing unfavourable conditions imposed by the very hot massive stars. The debate about on-going star formation in the region took a new dimension with the detection of proplyds-like objects that could imply on-going active low and intermediate mass star formation (see Dufour et al. 1998; Smith et al. 2003). Indications of on-going star formation became much more numerous in the last decade (Smith 2006). Very recently, an isolated neutron star candidate discovered in the neighbourhood of $\eta$ Car suggested the existence of at least two episodes of massive-star formation (Pires et al. 2009; Hamaguchi et al. 2007). 
Along with the Cygnus region, Carina is one of those historical fields where the X-ray emission linked to massive stars has been first discovered at the epoch of the EINSTEIN observatory (Seward et al. 1979). Indeed, this domain of wavelength is crucial for the study of several kinds of astronomical sources. In particular, massive OB stars exhibit intrinsic X-ray emission (Berghöfer et al. 1996, 1997). In addition to the two intrinsic emissions of their components, the massive binary systems characterized by important mass-losses may generate a third component of X-ray emission linked to a zone of collision between the two winds (Stevens et al. 1992).

Several authors suggested the possibility of detecting young low-mass pms objects through their hard X-ray emission which escapes the highly obscured regions (see Walter 1992; Kamata et al. 1997; Hofner \& Churchwell 1997; Hofner et al. 2002). Indeed, X-ray emission from low-mass pms stars is now a well established phenomenon (Neuhäuser 1997; Feigelson \& Montmerle 1999). These stars usually exhibit strong flares that are most probably due to powerful magnetic field reconnection events; these X-ray flares have characteristics that allow the identification of the star as a pms object. The X-ray emission process of pms stars has been interpreted as related to the corona, but new features (e.g. pms accretion, jets, outflows) have now been identified (see Chapters 2, 3 of Güdel \& Nazé 2009). X-rays are also known to be produced in high-mass starformation regions. The work of Hofner et al. (2002) suggests that the widespread X-ray sources in the W3 giant molecular cloud could be due to young ionizing stars embedded in ultracompact $\mathrm{H}$ II regions. As pointed out by Churchwell (2000), the hard $\mathrm{X}$-ray band could be the best wavelength domain to locate these young objects. Some examples of X-ray detected pms objects can be found in Garmire et al. (2000, Orion Nebula), in Gagné et al. (2004, $\rho$ Ophiuchi cloud); recent results on young open clusters seem to confirm the efficiency of the detection technique: see NGC 6530 (Rauw et al. 2002; Damiani et al. 2004) and NGC 6231 (Sana et al. 2006a,b, 2007). A recent review can be found in Feigelson et al. (2007).

Owing to the outstanding characteristics of the Carina region, it is therefore natural that it has been the subject of various studies and that astronomers decided to take the opportunity of the new generation of sensitive X-ray instruments represented by XMM-Newton and Chandra for performing systematic surveys for X-ray sources. Concerning XMM-Newton, the first new generation X-ray survey in the Carina region has been published by Albacete-Colombo et al. (2003) and was performed on the basis of two early observations of $\eta$ Carinae. Their study has been followed by the one of Antokhin et al. (2008) which merged the two $\eta$ Carinae pointings with three other ones centred on WR 25, a bright X-ray emitting WN star. This deeper survey covers a region about $30^{\prime}$ in diameter centred somewhere between $\eta$ Carinae and WR 25. It is remarkable that this X-ray survey detected no X-ray sources associated to the proplyd candidates. Some 242 sources are listed in this rich field. Concerning Chandra, the related studies are to be found in Evans et al. (2003, 2004) and Sanchawala et al. (2007). A deeper survey reaching lower-mass stars was also undertaken by Albacete-Colombo et al. (2008). The field centred on $\eta$ Car has also been observed recently with the Suzaku satellite (Ezoe et al. 2009) in order to conduct a study of the diffuse emission.

We here describe an X-ray survey of a circular (30' in diameter) field which is adjacent to that covered by Albacete-Colombo et al. (2003) and by Antokhin et al. (2008), and is situated west and slightly north of it. The field was observed several times while monitoring the orbit of WR 22, a massive WR+O binary.
Figure 1 locates the various X-ray surveys in the Carina region. Actually, WR 22 is situated further west of the western part of the V-shaped dark lane. We recall that WR 22 is supposed to be at a distance $D M=12.15(2.7 \mathrm{kpc}$, which agrees well with the open cluster distance given by Tapia et al. 2003) and is characterized by an excess $E_{B-V}=0.36$ (see Gosset et al. 2009). A catalogue of 43 bona-fide X-ray point sources is established and the first steps towards the identification of these sources are performed. Section 2 gives details on the observation and data reduction procedures. Section 3 explains the method used to detect the sources and the construction of the catalogue; it also provides the results of the cross-correlation with existing catalogues of objects. Section 4 presents a study of the variability in the X-ray domain of the various sources, while Sect. 5 deals with a few extracted X-ray spectra. Section 6 gathers a few comments and deductions about individual objects, while Sect. 7 deals with the diffuse X-ray component. Finally, we give a short conclusion in Sect. 8.

\section{Observations and data reduction}

The field of WR 22 (at $\alpha(\mathrm{J} 2000)=10^{\mathrm{h}} 41^{\mathrm{m}} 17^{\mathrm{s}} .5$ and $\delta(\mathrm{J} 2000)=-59^{\circ} 40^{\prime} 36^{\prime \prime}$ '9; $\equiv$ HD 92740) was observed with the XMM-Newton facility (Jansen et al. 2001) during seven pointings with nominal exposure times of $10 \mathrm{ks}$ (programme 010947). All pointings aimed at the study of the massive binary and were thus accurately centred on it. The corresponding results are described in a previous paper (Gosset et al. 2009). Here, we combined six of these pointings in order to perform a deep survey for X-ray sources situated in the Carina region around WR 22. The details of the six pointings are given in Table 1; the pointing labelled I in Gosset et al. (2009) was not used due to its brevity. The first column of Table 1 refers to an internal numbering of the pointings, while Cols. 2 and 3 give the orbit during which the observations were performed as well as the identification number of the pointing. The relevant observing date is to be found in Col. 4. We give the estimated duration of the observation in Col. 5, and in Cols. 6 to 8 the effective exposure times (soft proton flare corrected) for the three instruments. The last column provides the corresponding position angle (PA) of the satellite.

The two EPIC-MOS instruments were operated in the full frame mode (Turner et al. 2001) as was the EPIC-pn camera (Strüder et al. 2001). All three EPIC instruments needed the thick filter in order to reject the UV/optical light from the bright main target. We used version 5.3.3 of the XMM-Newton Science Analysis System (SAS) to reduce the raw EPIC data. We applied the emproc and epproc pipeline procedures to the EPICMOS and EPIC-pn raw data to generate proper event lists, taking particular care to preserve the adequation between the different pointings, both in translation and in orientation. The event lists were filtered in a standard way and cleaned for soft proton flares as described in Gosset et al. (2009). The resulting effective exposure times were reduced to the values given in Table 1, which also contains other relevant information. More details about the characteristics of the individual pointings can also be found in the paper of Gosset et al. (2009).

WR 22 is most of the time the brightest X-ray source in the field and is always at the centre. No pile-up was present in its image (Gosset et al. 2009). The pile-up limit is $6-8 \mathrm{cts} / \mathrm{s}$ for the pn detector, and the brightest source in the field reaches some $2 \%$ of this count rate. For MOS detectors, the limit is at $0.7 \mathrm{cts} / \mathrm{s}$ and the brightest source never exceeds $10 \%$ of this value. In addition, we scrutinised the (unfiltered) event lists for patterns in the range 


\section{Carina Region}

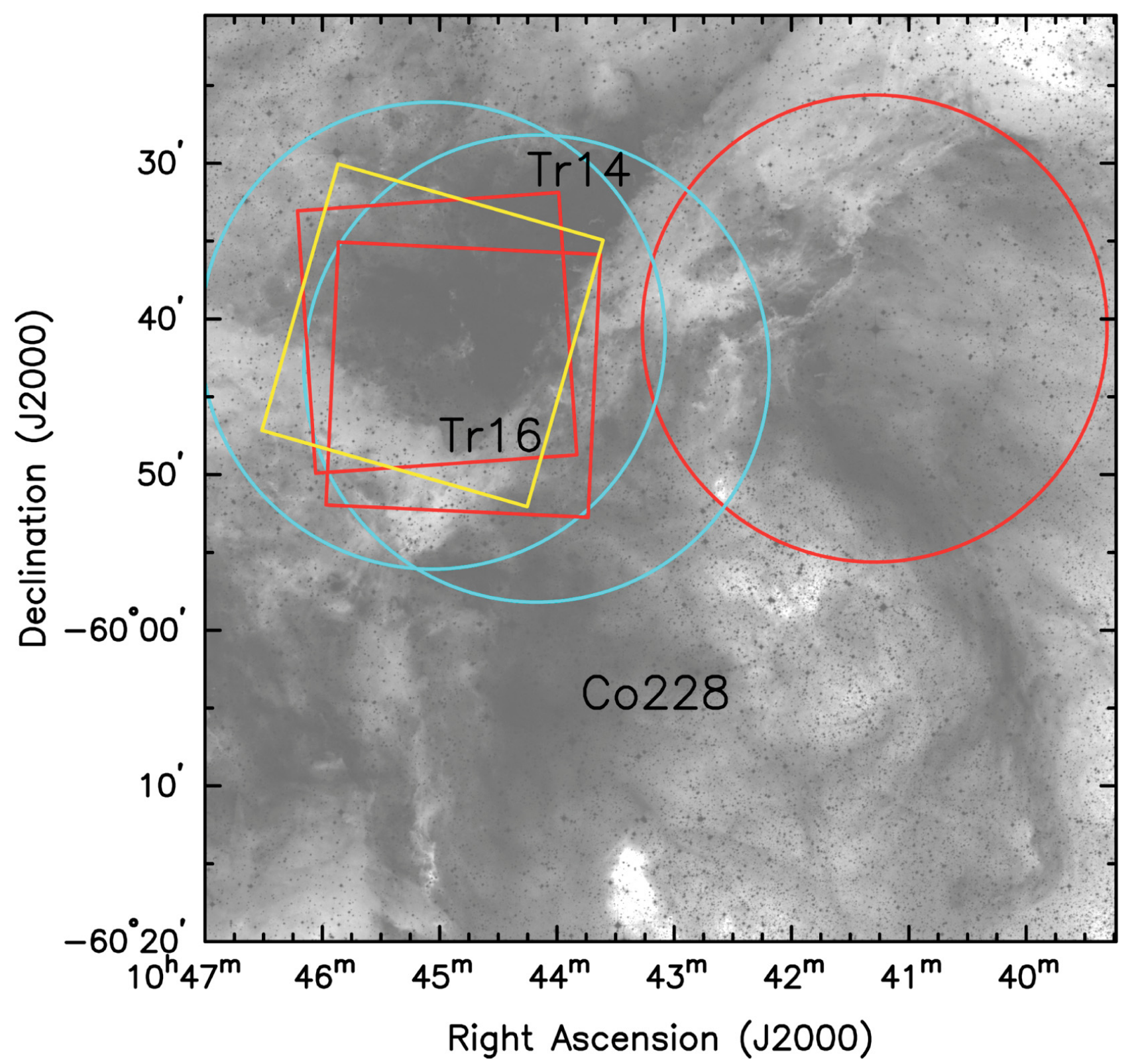

Fig. 1. Sketch of the Carina region with the positions of the various X-ray surveys. The regions are superposed to an $R$ filter image where the open clusters $\operatorname{Tr} 14$ and $\operatorname{Tr} 16$ are located. Starting from Tr 16, note the well-known V-shaped dark lane (appearing white). The field investigated here is on the right side, indicated by a circle (red in the electronic version of the paper, dark-grey in the printed version). The other two circles (light-blue, looking light-grey) represent the survey reported by Antokhin et al. (2008). Both surveys are based on XMM-Newton observations. The two squares (red/dark-grey) represent the Chandra pointings analysed by Evans et al. (2003, 2004) and Albacete-Colombo et al. (2008). The latter corresponds to the less tilted square. The third square (yellow/white) stands for the Suzaku investigation (Ezoe et al. 2009).

Table 1. Journal of the XMM-Newton observations used to perform the survey around WR 22.

\begin{tabular}{ccccccccc}
\hline \hline Dataset & Orbit & Proposal/Obs. ID & \multirow{2}{*}{$\begin{array}{c}\text { Date } \\
\text { (yyyy-mm-dd) }\end{array}$} & $\begin{array}{c}\text { Estimated duration } \\
(\mathrm{ks})\end{array}$ & \multicolumn{2}{c}{ Effective Exposure (ks) } & \multicolumn{2}{c}{ PA } \\
MOS1 & MOS2 & pn & $0^{\circ}:^{\prime \prime}$ \\
\hline II & 291 & 0109470201 & $2001-07-11$ & 13.0 & 12.4 & 12.4 & 9.1 & $315: 32: 04.5$ \\
III & 301 & 0109470301 & $2001-07-31$ & 13.0 & 12.3 & 12.3 & 9.0 & $332: 01: 21.2$ \\
IV & 304 & 0109470501 & $2001-08-06$ & 12.7 & 10.2 & 10.5 & 6.5 & $337: 06: 12.6$ \\
V & 307 & 0109470601 & $2001-08-13$ & 10.7 & 7.5 & 7.5 & 4.8 & $000: 42: 34.5$ \\
VI & 375 & 0109470701 & $2001-12-26$ & 12.8 & 12.2 & 12.1 & 8.6 & $120: 52: 46.3$ \\
VII & 379 & 0109471001 & $2002-01-02$ & 15.5 & 14.2 & 13.9 & 8.9 & $129: 44: 49.6$ \\
\hline Total & & & 77.8 & 68.8 & 68.8 & 46.9 & \\
\hline
\end{tabular}


26-29 (usually associated to pile-up), but none were found. Therefore, pile-up is never a problem here. We built images in three bandpasses $0.5-1.5 \mathrm{keV}$ (soft), $1.5-2.5 \mathrm{keV}$ (medium) and $2.5-10 \mathrm{keV}$ (hard), plus a total band $(0.5-10 \mathrm{keV})$ for each pointing and each instrument. The positions of the events were binned with a pixel size of 2 .'5. We also constructed the corresponding individual exposure maps. The pn exposure maps were corrected for the dead time of $6 \%$ (full frame mode). Note that the bandpasses defined here are different from those used in Gosset et al. (2009).

For each energy bandpass and for each instrument we combined the six pointings using the FTOOLS (version 5.0) task farith both on the images and on the exposure maps; this resulted in nine images and the same number of exposure maps. These nine images are our observational material. Figure 2 illustrates the investigated field and presents a three-colour image (combination MOS1+MOS2) that gives an idea of the softness or hardness of the various sources. Figure 2 corresponds to an image not corrected for the exposure map and thus still vignetted. The exposure time at the edge of the field is about one third of the central one. The various linear dark streaks visible in the figure correspond to the CCD gaps rotated according to the various pointings, each characterized by a particular position angle.

Some of the sources (see Sect. 5) are sufficiently bright to extract X-ray spectra for the different instruments and for the individual pointings. This was done in a way quite similar to what has been performed for WR 22 (Gosset et al. 2009, in particular their Sect. 3). However, to improve the signal, we tentatively extracted the spectra of constant objects on the basis of a combination of the individual pointings. Accordingly, we merged within the SAS, for each instrument, the cleaned total event lists issued from each of the six pointings into one combined event list; we did the same for the attitude files generated by the pipeline. On the other side, we built up individual spectra for each pointing and concomitantly constructed the ancillary response function (ARF) files using the task arfgen. In order to be able to later combine these ARF files, we used the redistribution matrix function (RMF) files provided by the ESA-SOC and not those generated by the SAS tasks. The various generated ARF files were combined through the FTOOLS task addarf to provide a generic ARF file for each instrument. The combined spectra were extracted, along with the background, by means of the task evselect applied to the relevant combined event lists. The final spectra were computed through the task grppha on the basis of the spectra extracted from the combined event lists from the combined ARF files and from the RMF files utilized to generate the individual ARF files. Spectra were binned to have a minimum of 15 and 5 counts per cell (for MOS and pn, respectively).

\section{X-ray sources in the field of WR 22}

Figure 2 clearly reveals various X-ray emissions from the field of WR 22. Several point sources are clearly visible, including WR 22, and a few hard sources are easily picked out (thanks to their blue colour). A ubiquitous diffuse X-ray emission is also particularly visible. We searched for X-ray sources on the basis of the nine deep (i.e. combining six pointings) images corresponding to the three instruments (MOS1, MOS2 and pn) and to the three energy bands (soft, medium, and hard). Basically, we applied two different methods of detection, which we describe below.

\subsection{Detection}

The first method is based on the classical use of the tools provided within the SAS (version 5.3.3). The task eboxdetect was first run in local detection mode and, after estimating the background maps with esplinemap, again in map-detection mode. The adopted detection-box size was 5 by 5 pixels, and the process was first performed for the 2'.5 basic pixels. Afterwards, it was run again at other scales with binned pixels with the resulting sizes of 5.'0 and of 7.'5. Visual inspection of the fitted background was necessary to prevent a possible bad behaviour of the splines. For each position on the image, a probability that the poissonian fluctuations of the estimated background give a number of counts at least as high as that observed is computed for each of the nine images. These probabilities are converted into logarithmic likelihoods through the formula

$L_{i}^{\prime}=-\ln p_{i}$

and are then combined into a total logarithmic likelihood by summation

$L_{\mathrm{tot}}^{\prime}=\sum_{i=1}^{i=9} L_{i}^{\prime}=-\ln \prod_{i=1}^{i=9} p_{i}$

This total logarithmic likelihood is of course dependent on the number of images and is transformed into a reduced logarithmic likelihood through the formula

$L_{\text {red }}=-\ln Q\left(9, L_{\text {tot }}^{\prime}\right)$,

where $Q=1-P$ and $P$ is the incomplete $\Gamma$ function. The decision whether or not there is a detection of a source can be taken in the classical way by fixing a lower threshold on $L_{\text {red }}$ above which the source is by convention considered to be real. A threshold in reduced logarithmic likelihood of 22 was chosen to avoid the contamination by many spurious sources detected in the high background (partly due to the diffuse X-ray emission) surrounding WR 22 while keeping all sources visible by eye on the total EPIC image; the detection process was repeated three times at different scales. Finally, 86 candidate sources were found at this stage.

The next task to be applied was emldetect which is based on a maximum-likelihood fitting of the theoretical local psf (point spread function) to the profile of the source candidate. The method is inspired by the work of Cruddace et al. (1988). The adjusted parameters are the positions on both axes and the intensity of the source. Because we performed this fitting in nine bands, it results in 11 free parameters. The logarithmic likelihood $L_{\mathrm{eml}}$ corresponding to the global fit is normalized to a $L_{2}$ logarithmic likelihood using the following formula

$L_{2}=-\ln Q\left(5.5, L_{\mathrm{eml}}\right)$.

A high value for $L_{2}$ implies a good representation of the observed profile by the expected local psf; we decided to classify as sources the objects associated to an $L_{2}$ above the critical threshold of $8.0^{1}$. The method mainly allows us to tag objects that have a profile incoherent with the instrumental one and either to reject them as sources or to apply further treatment. The method is also able to deal with an additional parameter representing the extent of the source. It is also able to adjust several blended sources simultaneously, although we never encountered this. Finally, this

1 All the likelihood values given in the present paper have been cor-
rected for the problem mentioned in the XMM-NEWTON NEWS \#29. 


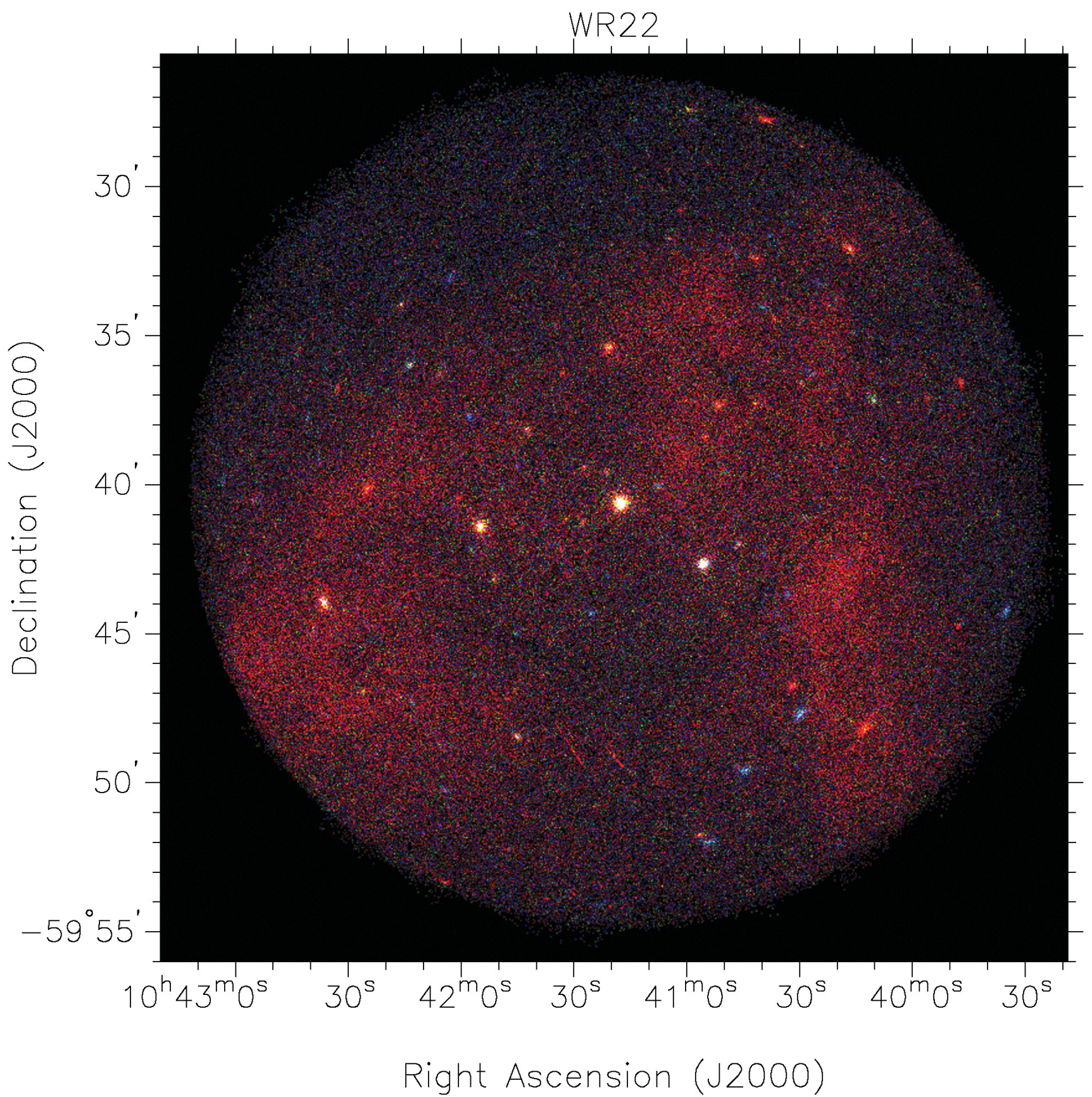

Fig. 2. Combined MOS1+MOS2 X-ray image of the field around WR 22 in the Carina region. The field is centred on this object at $\alpha(\mathrm{J} 2000)=10^{\mathrm{h}} 41^{\mathrm{m}} 17.5$ and $\delta(\mathrm{J} 2000)=-59^{\circ} 40^{\prime} 36^{\prime \prime} \cdot 9$. Shown is a false-colour image where the three colours red, green, and blue correspond to the soft, medium, and hard energy bandpasses. The full field of view is about $30^{\prime}$ in diameter.

reduced logarithmic likelihood $L_{2}$ of minimum 8.0 provides a refined set of 77 sources.

Those 77 sources were then checked individually by eye to remove objects generated by spurious spikes (five) or by mere fluctuations in the bright background (eight) and objects which were not detected significantly in at least two instruments (eight). Extended sources (thirteen) are further considered in Sect. 7 and were removed from the present data set. Our final list then contains 43 bona-fide X-ray point sources. They are listed in Table 2 and identified in the X-ray image by their number in Fig. 3.

In order to check our results, we tried a second detection method, running SExtractor (Bertin \& Arnouts 1996) with a detection threshold of $3 \sigma$ on the coadded EPIC image convolved beforehand with a Gaussian characterized by a full width at half maximum (FWHM) of 2 pixels. We also found 43 sources, among which 38 (i.e. 88\%) are in common with the previous list. The remaining five sources except one were present in the original set of 77 sources obtained with eboxdetect + emldetect. This further supports the list of Table 2 that thus constitutes our catalogue of X-ray sources. The second column gives the name of the source according to the rules defined by IAU.

The vignetting corrected count rates in the entire energy band (i.e. $0.5-10 \mathrm{keV}$ ) are listed in Table 2 for each instrument (Cols. 3 to 5) on condition that the relative error is smaller than 2 . The reported errors are those computed by emldetect on the three energy bands, except for extremely soft or hard sources detected in all three instruments, for which the errors are computed from 
WR22 field

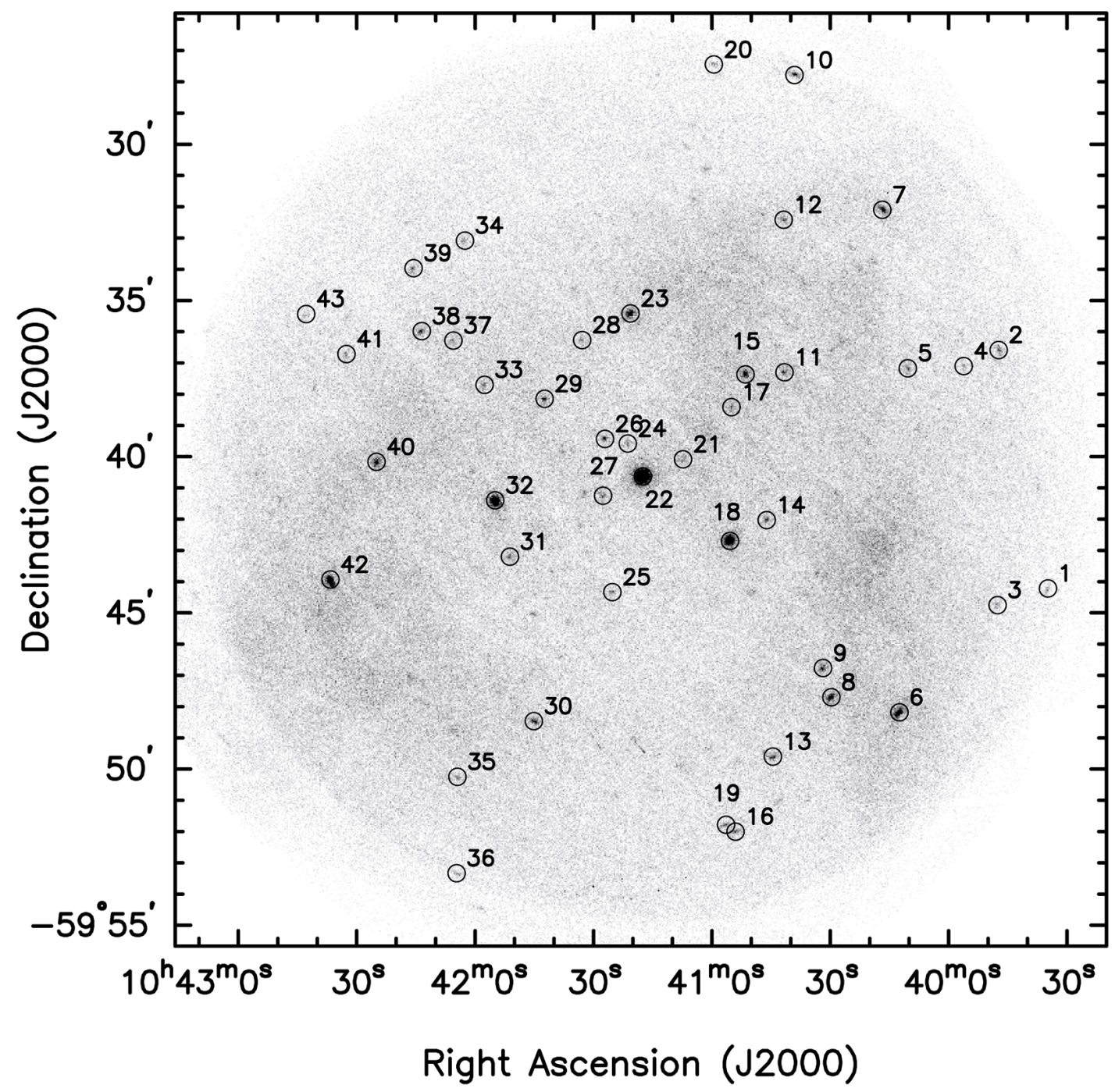

Fig. 3. Combined EPIC image (total band) of the WR 22 field based on the combined pointings II, III, IV, V, VI and VII. The 43 X-ray sources listed in Table 2 are identified by their number. The circles have a radius of $16^{\prime \prime}$, which corresponds to $70 \%$ encircled energy in the middle of the field.

only two bands: the soft and medium, or the medium and hard bands.

Finally, the hardness ratios defined by

$H R_{1}=\frac{\text { Medium }- \text { Soft }}{\text { Medium }+ \text { Soft }}$

and

$H R_{2}=\frac{\text { Hard }- \text { Medium }}{\text { Hard }+ \text { Medium }}$

are given for the pn (except when stated otherwise), provided the absolute error is $\leq 0.5(\sigma \leq 0.5)$. For extremely soft or hard sources, which are characterized by a huge count rate error in the hard or soft band, a value of $H R_{2}=-1$ or $H R_{1}=+1$ is attributed. Table 2 also gives the $\alpha(\mathrm{J} 2000)$ and $\delta(\mathrm{J} 2000)$ coordinates of each X-ray source (Cols. 8 and 9), the related formal positional error in arcsec $(1-\sigma$, Col. 10$)$ as well as the logarithmic likelihood $L_{2}$ (Col. 11), the estimated global background (total band, three instruments) at the source location (in counts per pixel, Col. 12) and the utilized cutting radius of the psf fit (Col. 13). This fit was performed up to this cutting radius, which roughly corresponds to $70 \%$ of encircled energy. Column 14 contains a variability flag (see Sect. 4).

The faintest detected sources of the combined pointings have psf and vignetting corrected count rates of about $2.5 \times 10^{-3} \mathrm{cts} / \mathrm{s}$ in pn and $1 \times 10^{-3} \mathrm{cts} / \mathrm{s}$ in MOS1 or MOS2. This rather high threshold is explained by a bright diffuse background and by the detection of many sources quite far away from the centre, where the effective exposure time is significantly lower.

The above procedure was also repeated for each individual pointing, except that the threshold of the reduced logarithmic likelihood in eboxdetect was chosen as $L_{\mathrm{red}}=15$ instead of $L_{\text {red }}=22$ to compensate for the lower background level. The task emldetect was then run with a minimum value of $L_{2}=6.25$. We then matched each individual source list with that obtained from the analysis of the combined image to identify possible new sources. No additional source was identified.

Several sources are not seen on each individual pointing and/or undergo count rate variations: this yields misleading mean values of the count rates in the catalogue because the latter are estimated on the basis of the total exposure time. These sources will be discussed in more detail in Sect. 4 . 
J.-F. Claeskens et al.: A search for X-ray sources in Carina

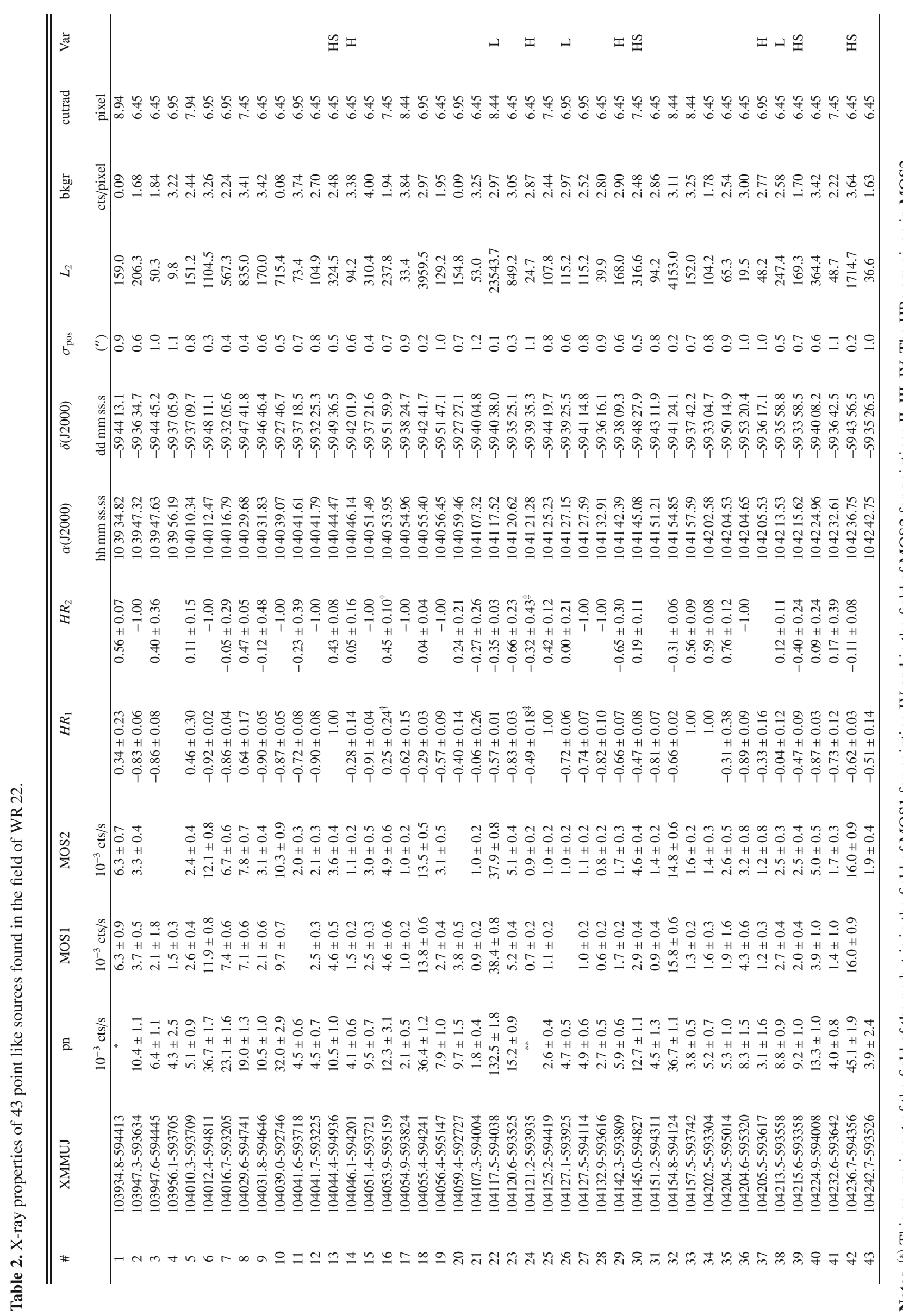

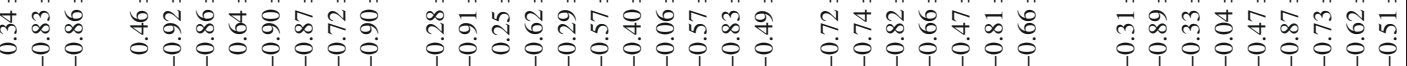

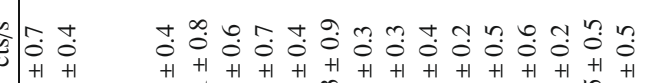

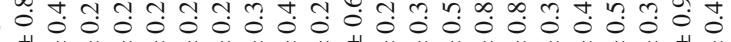

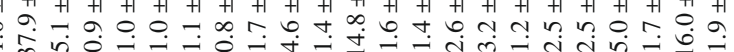



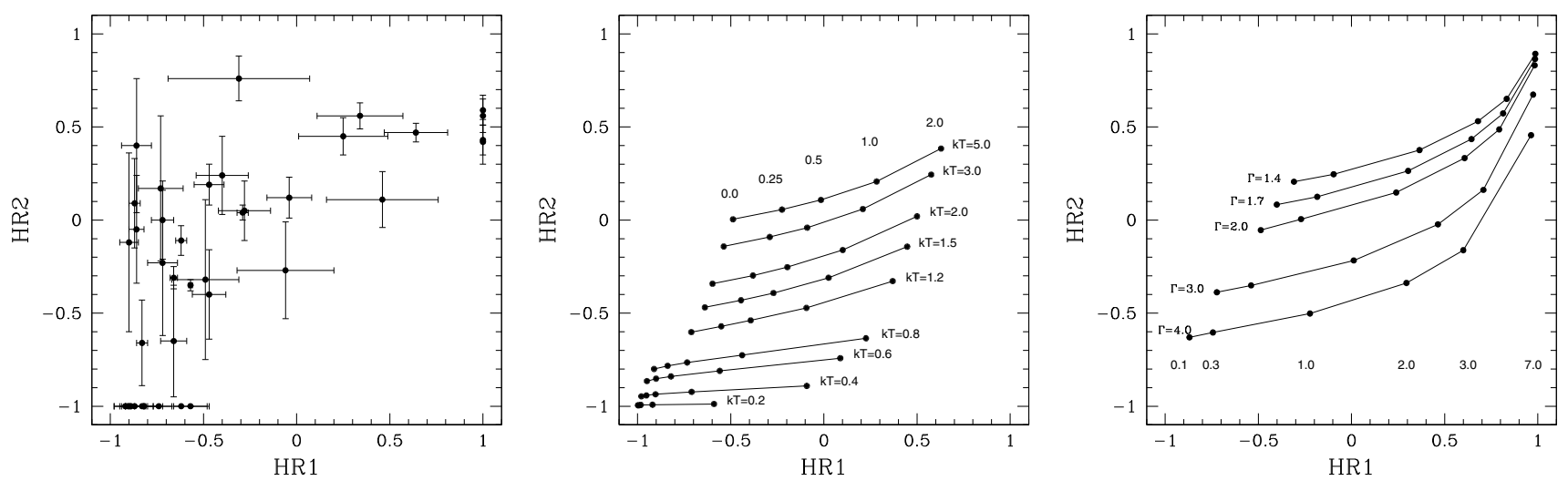

Fig. 4. Left panel: hardness-ratio diagram: $H R_{2}$ versus $H R_{1}$ for all the observed objects which have both values defined. The error bars represent one standard deviation. The four sources with $H R_{1}=1$ are from top to bottom \#34, \#33, \#13 and \#25. The four other hard sources are, in order of decreasing $H R_{1}, \# 8, \# 5, \# 1$ and \#16. Middle panel: computed positions in the same hardness-ratio diagram of synthetic on-axis sources (optically thin thermal plasma emission mekal models): solid lines represent the position of mekal models as a function of the absorbing column density $\left(N_{\mathrm{H}}=0 ., 0.25,0.5,1.0\right.$ and $2.0 \times 10^{22} \mathrm{~cm}^{-2}$, from left to right $)$, for several values of the temperature $(k T=0.2,0.4,0.6,0.8,1.2,1.5,2.0,3.0$ and $5.0 \mathrm{keV}$, from bottom to top). Right panel: idem but for powerlaw models $\left(N_{\mathrm{H}}=0.1,0.3,1.0,2.0,3.0\right.$ and $7.0 \times 10^{22} \mathrm{~cm}^{-2}$, from left to right), for several values of the photon index $(\Gamma=1.4,1.7,2.0,3.0$ and 4.0, from top to bottom $)$.

A faint X-ray source seems to be present at $\alpha(\mathrm{J} 2000)=$ $10^{\mathrm{h}} 42^{\mathrm{m}} 40^{\mathrm{s}} \cdot 6, \delta(\mathrm{J} 2000)=-59^{\circ} 37^{\prime} 42^{\prime \prime} \cdot 7$. It can be seen to the south east of source \#41 (Figs. 2 and 3). This possible source is at the limit of the detection. Actually, it is part of the eight candidates that were rejected because they were not present for at least two detectors. Indeed, the source is not detected and is hardly spotted by eye on the MOS1 and the pn images. Only the MOS2 detector leads to a detection in the combined dataset according to the above criteria. The source appears faint because it is only present in pointing $\mathrm{V}$ where it is detected by all three detectors. This is the most secure case among the eight rejected candidates, but we will not include it in our catalogue to be consistent with our procedure. This possible source is a good case in point of a faint limit object.

Figure 4 gives the hardness-ratio diagram $H R_{2}$ versus $H R_{1}$ for all sources which have both values defined. There is a wide variety of sources. Four objects (\#13, \#25, \#33 and \#34) have $H R_{1}=1.0$, which indicates hard X-ray sources. Four other sources (\#1, \#5, \#8 and \#16) are also quite hard, with both positive hardness ratios. Source \#38 has $H R_{2}>0$. but $H R_{1}$ is slightly negative. The eight hard objects indeed appear quite blue in Fig. 2 (also use Fig. 3). We computed synthetic hardness-ratios on the basis of simple spectral models. We first considered optically thin thermal plasma emission (mekal models, see Kaastra 1992; Mewe et al. 1985) of temperature $k T=0.2,0.4,0.6,0.8$, $1.2,1.5,2.0,3.0$ and $5.0 \mathrm{keV}$ with absorbing columns $N_{\mathrm{H}}=0$., $0.25,0.5,1.0$ and $2.0 \times 10^{22} \mathrm{~cm}^{-2}$. We also considered powerlaws with photon index $\Gamma=1.4,1.7,2.0,3.0$ and 4.0 accompanied by absorbing columns of $N_{\mathrm{H}}=0.1,0.3,1.0,2.0,3.0$ and $7.0 \times 10^{22} \mathrm{~cm}^{-2}$. The corresponding positions in the hardnessratio diagram are given in the middle and right panels of Fig. 4. We discuss the individual sources in Sects. 5 and 6.

\subsection{Comparison with the $1 X M M$ catalogue}

The field of WR 22 has been included in The XMM-Newton Serendipitous Source Catalogue: 1XMM. This catalogue contains 56711 X-ray sources, among which 33026 have a quality flag larger than 0 and a logarithmic likelihood larger than 8 (three EPIC instruments). In a 15 arcmin radius field centred on WR 22, 87 sources are found in the 1XMM catalogue, but only 54 have a total likelihood $>8$ and only 14 also have a quality flag equal to 4 , i.e. qualified as "good". Among the 43 sources listed in Table 2, 29 (i.e. 67\%) have a counterpart in the 1XMM catalogue within $20^{\prime \prime}$ of their position, among which 25 (i.e. $58 \%$ ) have a total logarithmic likelihood $>8$ and only 9 (i.e. $21 \%$ ) also have a quality flag equal to 4 . The apparent low completeness of the 1XMM partially arises because only the pointing II is used to build this part of the catalogue, while we used a deeper combined image.

Finally, regarding the measurements, we compared the count rates and the astrometry of the 1XMM sources that we also detect in pointing II (see Sect. 4 for details about the analysis of the individual pointings). Concerning the count rates, we confronted our results in the total energy band $0.5-10 \mathrm{keV}$ with those computed in the $0.5-12 \mathrm{keV}$ range from the individual energy bands listed in the 1XMM catalogue. As can be seen in Fig. 5, the agreement is excellent for the MOS1 and MOS2 instruments. However, our count rates are on average $7.9 \%$ brighter in the pn than those reported in the $1 \mathrm{XMM}$ catalogue. This is very probably due to the correcting factor of 1.06 that we introduced in the pn exposure maps to take into account the detector read-out dead time. After performing the same correction to the 1XMM count rates, the mean discrepancy with our results is reduced to $2.4 \%$ (see bottom graph in Fig. 5). The preliminary nature of the $1 \mathrm{XMM}$ catalogue is evident. Recently, the $2 \mathrm{XMM}$ catalogue has been released (see Watson et al. 2009). This catalogue is more extensive compared to the 1XMM, but still does not combine several pointings in the way we did in the present work.

\subsection{Optical and infrared counterparts}

The positions of the $43 \mathrm{X}$-ray sources found in the present XMM-Newton observations were cross-correlated with the Guide Star Catalogue (version 2.2: GSC2.2) ${ }^{2}$ and the Two Micron All Sky catalogue of Point Sources ${ }^{3}$.

\footnotetext{
2 The Guide Star Catalogue-II is a joint project of the Space Telescope Science Institute and the Osservatorio Astronomico di Torino.

3 2MASS is a joint project of the University of Massachusetts and the Infrared Processing and Analysis Center/California Institute of Technology, funded by the National Aeronautics and Space Administration and the National Science Foundation (see Cutri et al. 2003)
} 

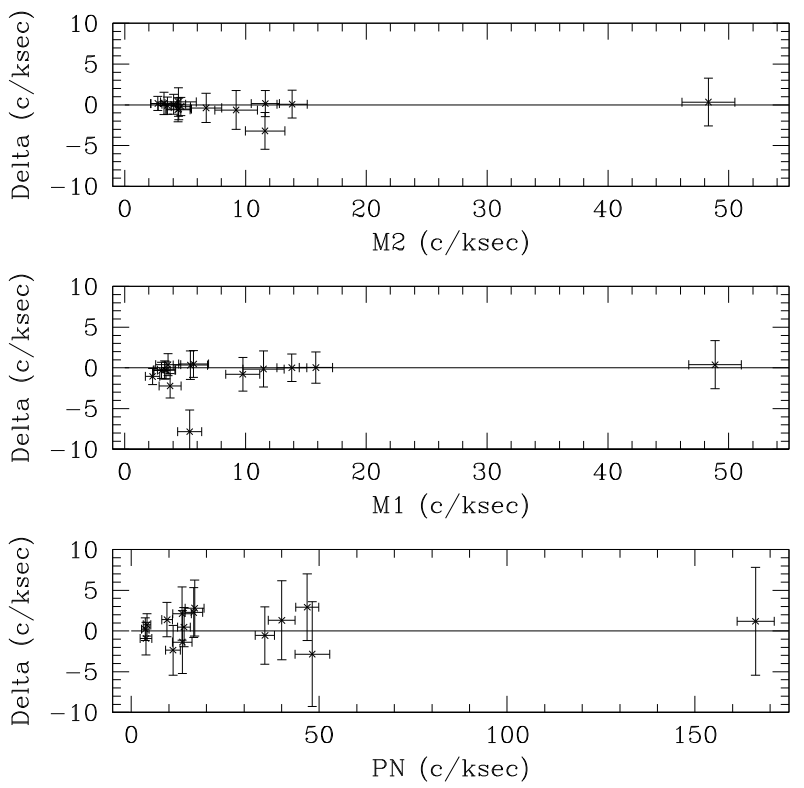

Fig. 5. Differences between measured count rates in pointing II and count rates from 1XMM as a function of the measured ones. For each instrument (MOS2: top; MOS1: middle; pn: bottom), sources in common and with meaningful count rate errors were selected. 1XMM count rates in pn were multiplied by the factor 1.06 .

In order to find a compromise between correlations missed due to astrometric errors and spurious associations in the crowded field of WR 22, we defined an optimal cross-correlation radius. We followed the approach described in Jeffries et al. (1997) by fitting to the observed cumulative number of crosscorrelations between an X-ray source and an optical/infrared counterpart as a function of the correlation radius, $\phi(\leq r)$, the formula

$$
\begin{aligned}
\phi(\leq r)= & A\left[1-\exp \left(\frac{-r^{2}}{2 \sigma^{2}}\right)\right] \\
& +(N-A)\left[1-\exp \left(-\pi B r^{2}\right)\right],
\end{aligned}
$$

where $N, A, \sigma$ and $B$ represent the total number of X-ray sources $(N=43)$, the number of true correlations, the uncertainty of the X-ray astrometry and the surface density of the existing catalogue with which the correlation is made.

From the run of the integrated number of correlations with the GSC2.2 catalogue as a function of the separation (42 X-ray sources have an optical source located closer than $\left.20^{\prime \prime}\right)$, we derived $A=35.4, B=2 \times 10^{-3} \operatorname{arcsec}^{-2}$ and $\sigma=11^{\prime \prime} 8$ (see also Fig. 6). We thus chose the optimal correlation radius $r_{\mathrm{o}}=5{ }^{\prime \prime}$, implying that the expected number of spurious associations among the 35 correlations listed in Table 3 is no more than 1. In other words, $79 \%$ of the detected X-ray sources in the field of WR 22 should be properly identified with an optical counterpart. Note that source \#28 is associated with a visible counterpart which turned out to be a GSC2.2 artefact and not an actual object. Therefore, we consider that source \#28 has no optical counterpart.

Concerning the infrared source catalogue, it is remarkable that the infrared counterparts closely match the position of most optical counterparts. The number of correlations XMM-2MASS with a small radius $\left(<3^{\prime \prime}\right)$ is higher than for correlations with the GSC2.2, as expected from the higher density of the 2MASS, but the fit of Eq. (5) is poor (see Fig. 6). It yields $A=40.35$ and $\sigma=1$." 6 , but to avoid unrealistically low values, the catalogue
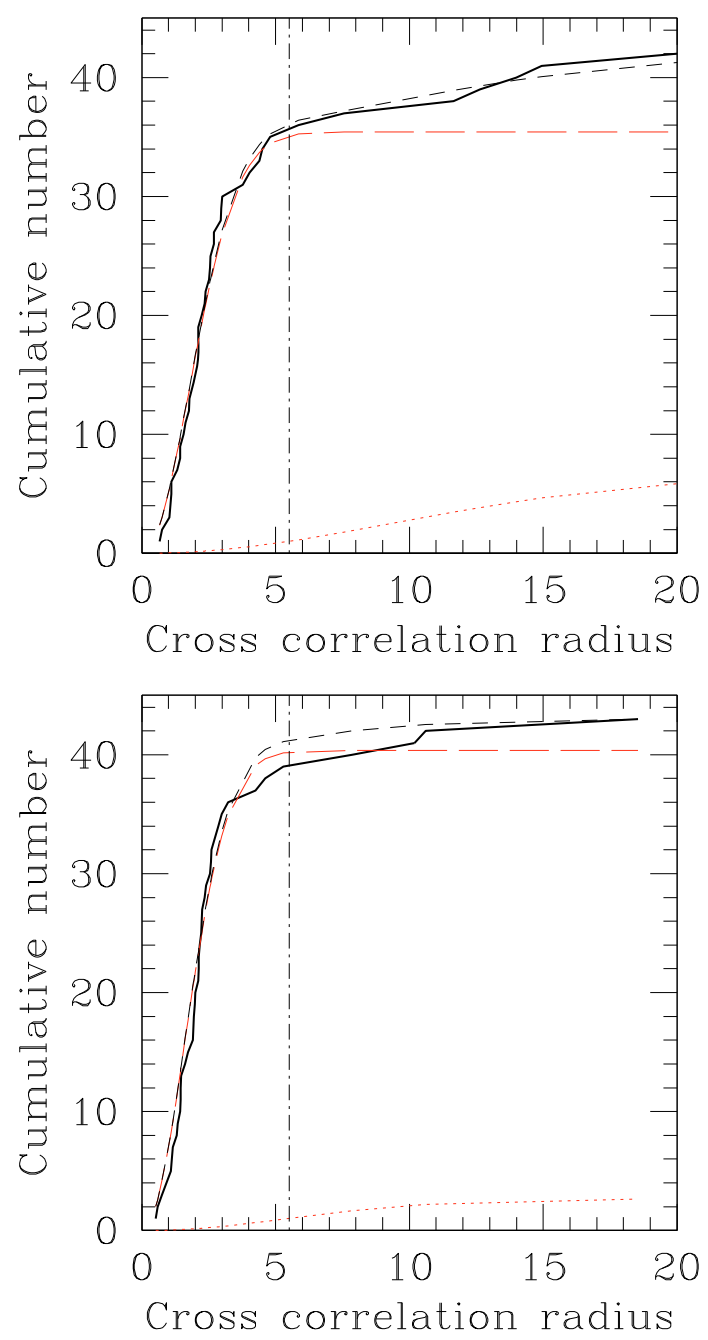

Fig. 6. Cumulative numbers of correlations between the X-ray detections and the GSC2.2 catalogue (upper panel) or the 2MASS catalogue (lower panel). The thick curves represent the observed numbers, the short dashed curves show the best fits and the dotted and long dashed curves correspond to the spurious and real number of correlations respectively. The vertical dot-dashed line identifies the optimal correlation radius at $55^{\prime \prime} 5$.

density had to be fixed from the infrared source count in the WR 22 field $\left(B=5 \times 10^{-3} \operatorname{arcsec}^{-2}\right)$. Finally, we decided to keep the same optimal radius $r_{\mathrm{o}}=55^{\prime \prime} 5$ as for the GSC2.2 catalogue, even if several multiple counterparts are found. However, note that four X-ray sources (\#1, \#18, \#21 and \#39) get an infrared counterpart with no optical counterpart, but the actual matching radius is much smaller than $r_{\mathrm{o}}$.

Table 3 shows the results of the cross-identification. The two first columns reproduce the numbering and the name of the $\mathrm{X}$-ray source in the catalogue. The following columns deal with the relation to the GSC2.2 catalogue. $n$ (Col. 3) is the number of counterparts, $d$ (Col. 4) the corresponding separation, and Col. 5 reports the star identified as the probable counterpart of the X-ray source. The three following columns report the $B, V, R$ photographic magnitudes taken from the GSC2.2. The next columns have a similar structure, but are related to the 2MASS catalogue. The three infrared magnitudes are those given in the catalogue and are in the natural system. They are followed by two triple flags giving an indication about the reliability of the three photometric measurements (see the 2MASS 
A\&A 525, A142 (2011)

in

急

.

.

(9)

을

$\approx$

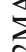

递

్ㅡㄹ

@)

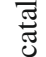

ป

है

$\frac{7}{2}$

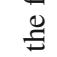

$\because$

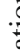
苞

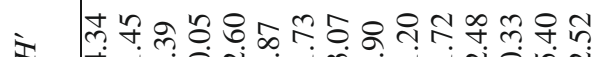

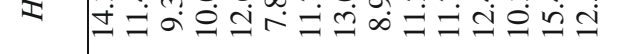

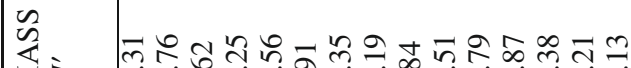

等

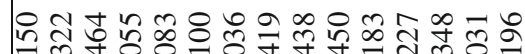

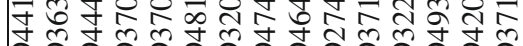

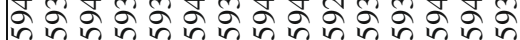

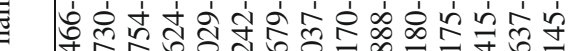

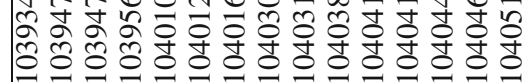

o

$=$

$\approx$

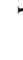

$\infty$

U.

号

$=$

$\tau \cong$

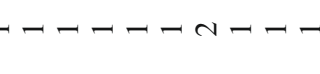

$\stackrel{n}{\simeq}$

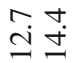

ํํ욤

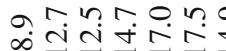

$\stackrel{\circ}{=} \vec{I} \quad \infty$

$\infty$

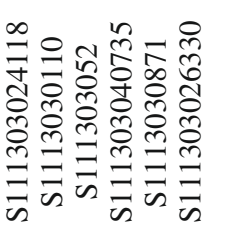

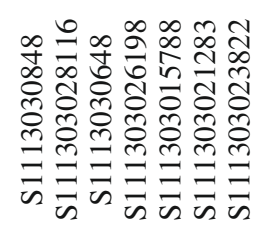

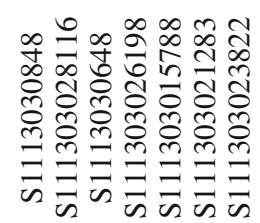

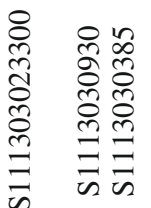

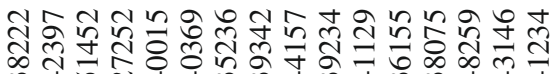

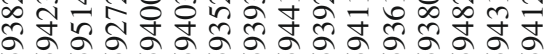

to 1 की

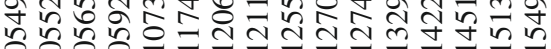

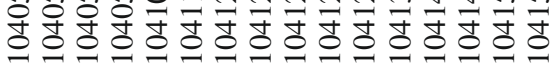

กู่

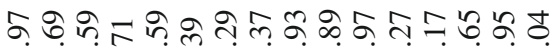

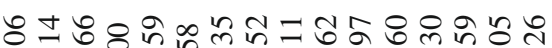

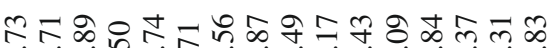

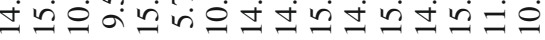

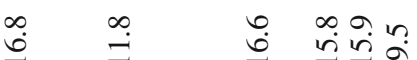

$\stackrel{2}{\stackrel{9}{3}}$

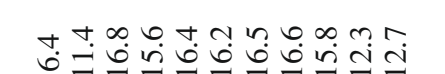
$\stackrel{n}{=}$

$m$

ì

in $\stackrel{\infty}{-}$

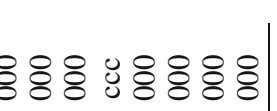

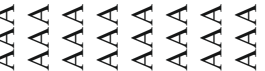

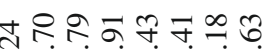

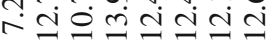

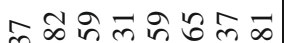

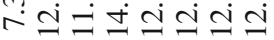

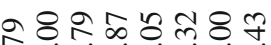

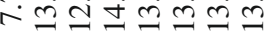

느요

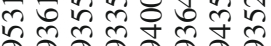

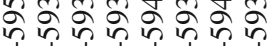

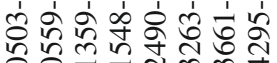

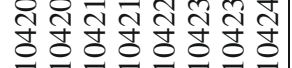

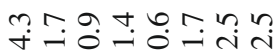

$-\neg-\neg-4-$

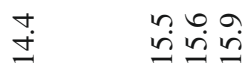

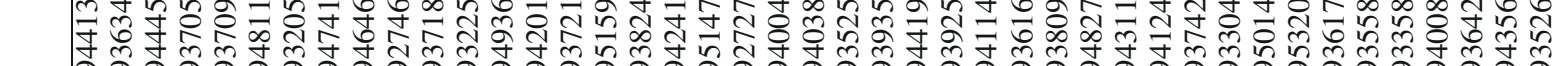

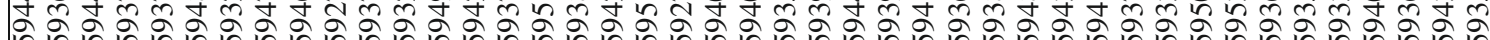

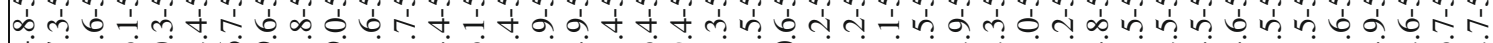

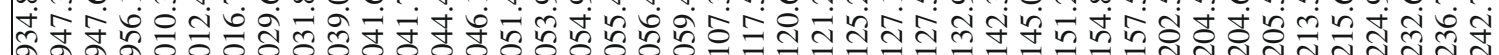

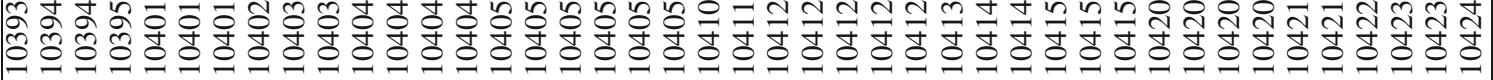
\# - - 
catalogue for a full interpretation of the flags). When several counterparts are identified, only the closest one is given.

In summary, we achieved a very good source identification. Indeed, 34 EPIC sources have a single true optical counterpart within 5 .'5 (their averaged correlation radius is $2 . .2 \pm 1$.'0) and only one of them (\#34) has no infrared counterpart. Note that sources \#28 and \#30 have a significantly closer infrared counterpart than their optical counterpart; this suggests that both counterparts are not related, which might thus be a sign of false association. We already know this to be true for source \#28. On the other hand, $34 \mathrm{X}$-ray sources have a single infrared counterpart, and five have two counterparts, but always with one of them within 2 .' 1 (the averaged correlation radius is $22^{\prime} 1 \pm 1$ '. 1 ). Four sources have no infrared counterpart. Only three EPIC sources (i.e. $7 \%$ ) have no counterpart at all. It is remarkable that among the eight hard sources $(\# 13, \# 25, \# 33$, \#34, \#1, \#5, \#8, \#16), four also miss an optical counterpart. One hard source (\#34) has no infrared counterpart. The positional errors deduced from the identification process are roughly a factor two larger than the formal ones reported in Table 2.

Finally, note that besides WR 22 itself, four detected EPIC sources are associated with bright known stars: \#6 = HD 92607 (spectral type O9II/III, Houk \& Cowley 1975) was already detected with the EINSTEIN observatory, see the Soft X-ray Source List (IPC: $11.5 \pm 2.4 \times 10^{-3} \mathrm{cts} / \mathrm{s}$; $\equiv 1 \mathrm{E} 1038.3-$ 5932; Chlebowski et al. 1989); \#9 = HD 92644 (spectral type O9.5/B0III, Houk \& Cowley 1975); \#19 = HD 305441 (spectral type A, Nesterov et al. 1995); \#36 = CD-59³233 (unknown spectral type). Source \#32 is close to HD 92836 but actually corresponds to an object that is $15^{\prime \prime}$ further south.

\subsection{Expected number of sources}

We estimate our detection limit to be around $2.5 \times 10^{-3} \mathrm{cts} / \mathrm{s}$ in the EPIC-pn for the range 0.5-10 keV. Motch et al. (2003) established counts of X-ray sources in the galactic plane. At the level of $2.5 \times 10^{-3} \mathrm{cts} / \mathrm{s}$, their $\log N-\log S$ relation (defined in the range $0.5-2.0 \mathrm{keV}$ ) predicts 139 sources per square degree, whereas at the limit of $1.7 \times 10^{-3} \mathrm{cts} / \mathrm{s}$ (value roughly corrected for the difference in the considered energy domain), it gives 225 . This would correspond to 27 and 44 sources respectively for the present survey. The first value is certainly to be considered as a lower limit.

We can also estimate the number of extragalactic background sources. To give a rough first order estimate of the total galactic extinction towards our line of sight, we made use of the DIRBE/IRAS maps provided by Schlegel et al. (1998). It must be clear that caution is necessary for using these maps near the galactic plane (Schlegel et al. 1998). In addition, it is well known that the extinction towards Carina is rather patchy and may vary by large amounts. In the region of WR 22, the colour excess $E_{B-V}$ varies from 3 to 16 . Using the gas-to-dust-ratio study of Bohlin et al. (1978), we convert these colour excesses to a hydrogen column density between 1.7 and $9.3 \times 10^{22} \mathrm{~cm}^{-2}$. More recent values of the conversion factor remain in good agreement with the one we adopted (Ryter 1996; Vuong et al. 2003). If we admit that background sources are mainly AGN with a powerlaw energy distribution characterized by a photon index $\Gamma=1.4$, we can deduce unabsorbed fluxes ranging from 0.57 to $1.2 \times$ $10^{-14} \mathrm{erg} \mathrm{cm}^{-2} \mathrm{~s}^{-1}$ in the $0.5-2.0 \mathrm{keV}$ range and 1.6 to $3.6 \times$ $10^{-14} \mathrm{erg} \mathrm{cm}^{-2} \mathrm{~s}^{-1}$ in the $2.0-10 \mathrm{keV}$ range. The first value corresponds to a low extinction, the second to a high extinction. Using both the soft and hard domain $\log N-\log S$ relation of Giacconi et al. (2001), we predict from 15 (high extinction) to 30 (low extinction) background sources in the surveyed field. Since the high extinction is expected for about half of the surface of the field, we estimate the amount of background sources to be around 20. These figures allow us to derive rough proportions for each kind of objects. At least $57 \%$ and up to $69 \%$ of the X-ray sources must be galactic in origin. The exact value could be higher because we are looking at a spiral feature. Indeed, $79 \%$ of the X-ray sources (i.e. 34) have a counterpart, which further supports this idea. The total number of background sources could be slightly overestimated, most probably due to the important background preventing the detection of faint objects (see Sect. 7).

\section{Variability of the X-ray sources}

The individual pointings are spread over a period of about 6 months, between July 2001 and January 2002 (see Table 1). As mentioned above, they were analysed individually. Therefore, this is a unique opportunity to look for a longterm variability of the count rates from pointing to pointing. Identifying significantly variable sources is also important to correct their averaged count rates listed in Table 2 into more relevant instantaneous count rates.

\subsection{Long-term variability}

For each individual pointing (II to VII), we ran the emldetect task, feeding it with the eboxdetect output source list obtained on the combined image in Sect. 3.1. We used a lower value of the minimum threshold $\left(L_{2}=5\right)$ in order to include all sources detected in the combined image. Then the individual measurements were extracted for the 43 sources matching the list given in Table 2. Finally, we checked the significance of the detections in each energy band for each instrument and recomputed the total error bars from the valid detections when necessary. Sources for which a significant variability was noticed were inventoried and flagged in Table 2 (column labelled Var).

Sources \#26 and \#38, as well as WR 22 itself undergo rather smooth but significant count rate fluctuations, as illustrated in Fig. 7. These are considered as long-term variables $(L)$, although \#26 deviates essentially in one single pointing.

On the opposite, the other variable sources do only show up in all instruments at one or two individual epochs and remain undetected the rest of the time. Their measured (instantaneous) count rates, given in Table 4, are significantly different from the averaged ones listed in Table 2. Among these objects listed in Table 4 (labelled H, for high state), sources \#30, \#39 and \#42 are becoming remarkably bright, the latter is even brighter than WR 22 in dataset VII. These objects are rather variable sources that exhibit bursts. Because of its behaviour, we also give the information for source \#26, although this star is detected even in its low state.

\subsection{Short-term variability}

We also extracted the event lists associated with each source in each dataset and built the intra-pointing lightcurves to check for short-term variability.

The source counts used for the lightcurves were considered in the energy range $0.5-10 \mathrm{keV}$ and within fixed circular, 13" radius apertures. The background was estimated in the same energy range within annuli defined between $13^{\prime \prime}$ and $21^{\prime \prime}$. Concerning the pn detector, the background was estimated from time to time in a nearby area chosen to lie on the same CCD. 

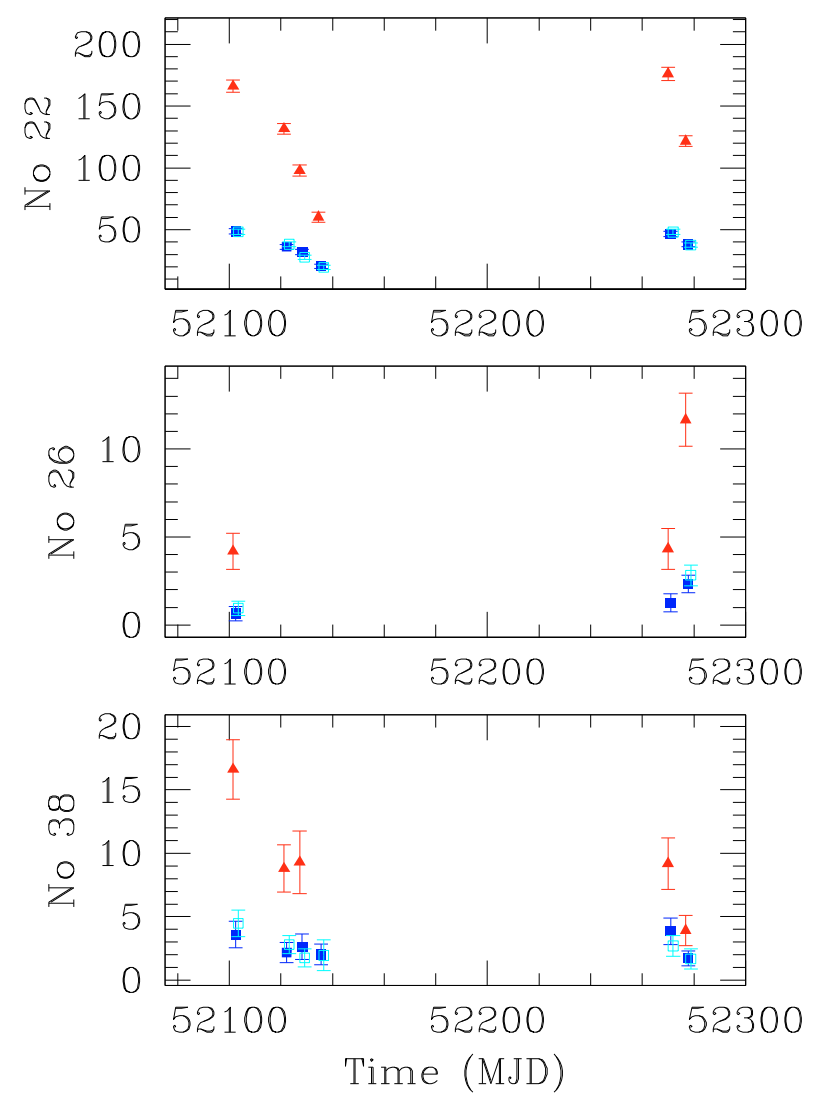

Fig. 7. Long-term count rate variations of sources \#22 (WR 22), \#26 and \#38. Measurements are made in the total energy band $(0.5-10 \mathrm{keV})$, in pn (solid triangles), MOS1 (solid squares) and MOS2 (open squares). The count rates are given in units of $10^{-3} \mathrm{cts} / \mathrm{s}$. In the same units, the subtracted background amounts to $0.3-1.0$.

Table 4. Count rates in the full energy range $0.5-10 \mathrm{keV}$ of variable sources exhibiting bursts and thus only seen in some individual datasets.

\begin{tabular}{ccccc}
\hline \hline Source & Dataset & \multicolumn{3}{c}{ Count rate $\left(10^{-3} \mathrm{cts} / \mathrm{s}\right)$} \\
$\#$ & & pn & MOS1 & MOS2 \\
\hline 13 & VI & $19.4 \pm 3.6$ & $9.1 \pm 1.7$ & $5.5 \pm 1.2$ \\
13 & VII & $18.0 \pm 2.3$ & $8.7 \pm 1.6$ & $6.5 \pm 1.2$ \\
14 & IV & $13.7 \pm 1.9$ & $5.0 \pm 0.9$ & $4.2 \pm 0.9$ \\
14 & V & $15.1 \pm 2.5$ & $4.2 \pm 1.2$ & $1.7 \pm 0.8$ \\
24 & VII & $3.6 \pm 2.3$ & $1.1 \pm 0.8$ & $1.6 \pm 0.5$ \\
29 & VII & $15.9 \pm 1.9$ & $5.1 \pm 0.8$ & $4.9 \pm 0.9$ \\
30 & III & $44.6 \pm 3.4$ & $17.4 \pm 1.7$ & $23.1 \pm 2.1$ \\
37 & IV & $14.2 \pm 2.5$ & $4.1 \pm 1.1$ & $6.3 \pm 1.3$ \\
39 & II & $40.0 \pm 3.6$ & $11.5 \pm 1.7$ & $11.6 \pm 1.6$ \\
42 & VII & $165.8 \pm 7.7$ & $49.5 \pm 2.9$ & $52.2 \pm 3.0$ \\
\hline 26 & VII & $11.7 \pm 1.5$ & $2.3 \pm 0.5$ & $2.8 \pm 0.6$ \\
\hline
\end{tabular}

Notes. Source \#26 is appended to the list for information but is not considered as a member (see end of Sect. 4.1).

Three statistical procedures were applied to test the null hypothesis of non-variability:

1. The Kolmogorov-Smirnov test was used to check whether the cumulative number of detected photons in the source as a function of time is actually compatible with that expected from a constant source, once the good time intervals are selected. For a deviation towards variability, the same test was applied on the background to insure that the deviation is only present for the object and that the signal is thus real.
2. The $\chi^{2}$ test was used to check whether the binned, background-corrected source counts are compatible with the constant averaged value obtained during the whole integration time. The bin size is chosen to be $200 \mathrm{~s}$ except when locally corrected to account for the absence of good time intervals.

3. The probability of variability (pov) test (Preibisch \& Zinnecker 2002; as revisited by Sana et al. 2004) was also applied.

Variability in one instrument is detected when the null hypothesis is rejected with a probability higher than $99 \%$ by at least $t w o$ of the above-mentioned tests. Variability of a source is established if it is detected in at least two instruments (except when only one instrument can be considered).

Significant short-term variability (labelled S) is found only among the sources exhibiting bursts already identified in Table 4 as only detected in a few individual datasets. Indeed, sources \#13, \#30, \#39 and \#42 (i.e. 50\%) are found to be variable during the particular pointing. No signatures of flares are found, which is not unexpected given the relatively short individual exposure times. However, long flares are not excluded as an explanation for the epoch to epoch fluctuations of these sources (in particular source \#42).

\section{X-ray spectra for a few sources}

A few sources in the studied field are sufficiently bright to permit the extraction of a useful X-ray spectrum. Some of the sources dominate in only one pointing and are much fainter or even undetected during the other pointings. For these sources, the extraction of the spectrum is only useful for the single pointing where they appear brighter. On the opposite, a few constant sources only have sufficient counts in the combined image so that an interesting spectrum can only be extracted from the combined event lists. Therefore, we will treat these two families separately.

The spectrum extraction technique is described in Sect. 2. We used the XSPEC software (version 11.0.1; see Arnaud 1996, 1999) in order to study the extracted spectra. These spectra were analysed by fitting various models, basically absorbed powerlaw or absorbed optically thin thermal plasma emission model (i.e. mekal, Kaastra 1992; Mewe et al. 1985), and in particular one-temperature (1-T) and two-temperature (2-T) models. A word of caution is necessary here: the resulting fits as given below are basically formal and indicative. The low quality of the spectra, which is due to the faintness of the sources, implies that the best-fit model does not necessarily represent the physical truth. For example, a 2-T mekal model fitting the data very well does not mean that we have to deal with two different plasmas at two different temperatures, but could rather suggest a plasma exhibiting a range of temperatures. In some cases, a successful 1-T model is the mere reflection of a lack of information (see Sect. 4.1 of Sana et al. 2006b). It would be tedious to detail all the models we tentatively fitted. We restricted ourselves to give the simplest, formal, best-fit results. Also, we adopted the solar abundances of Anders \& Grevesse (1989) motivated by a lack of further information, or at least of contradictory information; again the quality of the data prevented us in most cases to refine the analysis more. However, we also performed the various fits with abundances 0.3 solar as a check. In general, this had no significant impact on the resulting fit except concerning the emission measures. When the change has a significant impact, the alternative solution is also mentioned. 


\subsection{The single $X$-ray spectra}

A few objects (\#7, \#30, \#39 and \#42) were sufficiently bright during one pointing (typically, more than 120 actual counts in pn, 75 counts in MOS). The latter three are clearly variable, thus explaining the importance of one particular pointing. Object \#7 appears brighter in pointing IV, particularly in the pn detector, but this object has not been statistically recognized as variable. Spectra were extracted for each of these objects and fitted with various models. We report the best fits, which usually correspond to models of the kind wabs*mekal+mekal. We further tried models of the kind wabs*mekal+wabs*mekal. Usually, the hot component does not dominate in the soft part of the spectrum and thus the absorbing column density in front of it is little constrained. It can often be set to zero. Therefore, from the fit point of view, the hot component does not need an absorbing column. The above-mentioned first model is systematically retained in order to allow us to better compare the different spectra. The three spectra corresponding to the three EPIC instruments were fitted simultaneously. We recall that WR 22, which we associate with the Carina region, is characterized by $D M=12.15$, $A_{V}=1.12, E_{B-V}=0.36$ leading to (according to Bohlin et al. 1978) $N_{\mathrm{H}}=0.25 \times 10^{22} \mathrm{~cm}^{-2}$ (see Gosset et al. 2009). The extinction towards this region and beyond is however believed to be particularly patchy.

Table 5 summarizes the results of the fits. It is organised in two parts corresponding to Sects. 5.1 and 5.2. The first column gives the internal number of the object, whereas the second column indicates the pointing where it dominates. For constant objects, the second column recalls that the spectra were extracted from the combined event lists. The third column indicates the instrument in use (MOS: the two MOS detectors; pn: the pn alone; EPIC: all three instruments). Columns 4 to 6 give, respectively, the absorbing column density in front of the cooler plasma, the temperature of this cooler component and the temperature of the hotter one. Column 7 gives the reduced $\chi^{2}$ and the number of degrees of freedom (d.o.f.) associated to the fit. Column 8 shows the observed X-ray flux as deduced from the model in the energy range $0.5-10 \mathrm{keV}$. The numbers to the right of the fitted values represent the lower and upper borders of the range of the variations of the parameters corresponding to a $90 \%$ confidence level on one parameter $\left(\Delta \chi^{2}=2.706\right)$. The last two columns indicate the counts in the observed spectra and some useful remarks.

\subsection{The combined $X$-ray spectra}

For a few bright sources (typically, more than 500 actual combined counts in pn, 300 in MOS; i.e. \#6, \#18,\#23 and \#32) which were not suspected of variability, we combined the event lists of the different pointings, to improve the signal-to-noise ratio in the computed spectra. These X-ray spectra were analysed in a similar way as in Sect. 5.1, but the two MOS detectors and the pn one were fitted separately. As can already be deduced from Table 2 and Fig. 4 (and Fig. 2), source \#6 is quite soft. For reasons that will become clear in Sect. 6 , we tested whether a column $N_{\mathrm{H}}=$ $0.25 \times 10^{22} \mathrm{~cm}^{-2}$ (as for WR 22) is still acceptable: the results with a column fixed to that value are also given in Table 5.

Object \#32 falls quite often close to a CCD gap on the pn detector and is only safely distant from such a gap for pointings II and V. Therefore, the combination of the event lists to build a spectrum for the pn is a vain effort and we restricted our combined spectra to the two MOS detectors.

\section{Discussion and notes on individual objects}

The field surveyed here for X-ray sources has never been covered by a deep photometric survey like those performed in the neighbouring fields. We have thus no information to help the identification process. Although we have plans to remedy this, we have in the meantime to rely on the 2MASS photometry. An infrared colour-colour diagram of the type $J-H, H-K$ is a useful classification tool even if the information remains partial. We transformed the 2MASS $J^{\prime}, H^{\prime}$ and $K_{\mathrm{s}}^{\prime}$ magnitudes of the infrared counterparts into the standard $J, H, K$ ones. This was done using the updated version of the colour transformations of Carpenter ${ }^{4}$ (2001). The $J-H$ versus $H-K$ diagram for the X-ray source counterparts is given in Fig. 8. In the same diagram we also traced the expected loci of normal, unreddened main sequence and giant stars (taken from Bessell \& Brett 1988), as well as the reddening band for normal dwarves adopting the law derived by Rieke \& Lebofsky (1985). In addition, we give the expected position of the unreddened classical T Tauri stars as computed by Meyer et al. (1997).

Among the five hard sources present in Fig. 8 (squares), four (\#1, \#5, \#8 and \#13) appear to be reddened (located around $(J-H, H-K)=(1.1,0.4))$, only source \#25 appears near the main sequence branch at $(J-H, H-K)=(0.43,0.15)$. Below, we use Fig. 8 as a support to the discussion of a few individual interesting objects.

\subsection{Source \#6}

It is located at $(J-H, H-K)=(0.09,-0.05)$ in Fig. 8 and clearly points to an early-type star at the transition O-B. Indeed, the X-ray source \#6 is correlated with HD 92607, which is classified O9II/III (Houk \& Cowley 1975). This corresponds to $(B-V)_{0}=-0.31$ (Schmidt-Kaler 1982). The observed value $B-V=0.0$ (Table 3 ) suggests an excess $E_{B-V}=0.31$. This is of the order of the excess adopted for WR $22\left(E_{B-V}=0.36\right)$ that we associate with the Carina region. If we adopt $D M+A_{V}=12.15+$ $1.12=13.27$, as for WR 22, we have $V-M_{V}=13.27$, which for $V=8.23$ (Forte 1976) yields $M_{V}=-5.05$ in good agreement with an O9III star (Howarth \& Prinja 1989) in the Carina region. Dereddening the X-ray flux $f_{\mathrm{X}}=2.0-2.4 \times 10^{-14} \mathrm{erg} \mathrm{cm}^{-2} \mathrm{~s}^{-1}$ for the sole interstellar column gives at most an unabsorbed flux $f_{\mathrm{X}}^{\text {un }}=4.6 \times 10^{-14} \mathrm{erg} \mathrm{cm}^{-2} \mathrm{~s}^{-1}$ which gives $L_{\mathrm{X}}=4 \times 10^{31} \mathrm{erg} \mathrm{s}^{-1}$ $\left(\log L_{X}=31.6\right)$, leading to $\log L_{X} / L_{\text {bol }}=-7$. This value is about the expected one for a single O star (Berghöfer et al. 1997; Sana et al. 2006b). The star was previously known as an X-ray source observed by EINSTEIN (1E1038.3-5932). The derived plasma temperature of $0.6 \mathrm{keV}$ is rather typical of an $\mathrm{O}$ star, which further supports the identification.

\subsection{Source \#9}

It is associated to HD 92644 (O9.5/B0III, Houk \& Cowley 1975). This star is also a bright object $(V=8.84$, Forte 1976), has visible colours similar to HD 92607 (Forte 1976) and is located in the early-type star domain in Fig. $8((J-H, H-K)=$ $(-0.01,-0.09))$. If we assume that it lies at the same distance (same reddening) as HD 92607, a luminosity class V or IV would be preferred to III when the absolute magnitude $\left(M_{V}=-4.4\right)$ is considered. The observed X-ray count rate is

\footnotetext{
4 http://www.ipac. caltech.edu/2mass/releases/allsky/ doc/sec6_4b.html
} 
A\&A 525, A142 (2011)

Table 5. X-ray spectrum analysis: best-fit 1-T or 2-T models of the type wabs*mekal or wabs*mekal+mekal.

\begin{tabular}{|c|c|c|c|c|c|c|c|c|c|}
\hline Source & Pointing & Instrument & $\begin{array}{c}N_{\mathrm{H}} \\
\left(10^{22} \mathrm{~cm}^{-2}\right)\end{array}$ & $\begin{array}{c}k T_{1} \\
(\mathrm{keV})\end{array}$ & $\begin{array}{c}k T_{2} \\
(\mathrm{keV})\end{array}$ & $\chi_{v}^{2}$ (d.o.f.) & $\begin{array}{c}f_{\mathrm{X}}(0.5-10 \mathrm{keV}) \\
\left(10^{-14} \mathrm{erg} \mathrm{cm}^{-2} \mathrm{~s}^{-1}\right)\end{array}$ & Counts & Remarks \\
\hline \#7 & IV & EPIC & $0.53_{0.00}^{0.87}$ & $0.25_{0.14}^{0.79}$ & $2.88_{1.12}^{7.5}$ & $0.66(24)$ & 8.3 & 269 & see (1) \\
\hline \#30 & III & EPIC & $0.19_{0.06}^{0.41}$ & $4.03_{2.66}^{0.83}$ & - & $1.03(42)$ & 14.0 & 310 & see (2) \\
\hline \#39 & II & EPIC & $0.22_{0.00}^{0.50}$ & $0.90_{0.72}^{1.200}$ & $9.54_{3,43}^{-}$ & $1.19(19)$ & 10.3 & 292 & see (3) \\
\hline \#42 & VII & EPIC & $0.76_{0.00}^{1.46}$ & $0.61_{0.17}^{1.1 .2}$ & $3.62_{2.97}^{4.63}$ & $0.97(104)$ & 32.7 & 1240 & see (4) \\
\hline$\# 42$ & VII & EPIC & $0.94_{0.00}^{1.46}$ & $0.37_{0.08}^{1.22}$ & $3.08_{2.32}^{4.25}$ & $0.97(104)$ & 32.7 & 1240 & 0.3 solar \\
\hline \#6 & Comb. & MOS & $0.15_{0.00}^{0.33}$ & $0.63_{0.55}^{0.69}$ & - & $1.26(113)$ & 2.4 & 674 & \\
\hline \#6 & Comb. & $\mathrm{pn}$ & $0.11_{0.00}^{0.30}$ & $0.60_{0.54}^{0.69}$ & - & $1.01(63)$ & 2.0 & 633 & \\
\hline \#6 & Comb. & MOS & $0.25^{*}$ & $0.61_{0.53}^{0.54}$ & - & $1.27(114)$ & 2.3 & 674 & \\
\hline \#6 & Comb. & $\mathrm{pn}$ & $0.25^{*}$ & $0.49_{0.31}^{0.63}$ & - & $1.01(64)$ & 2.0 & 633 & \\
\hline \#18 & Comb. & MOS & $1.08_{076}^{2.02}$ & $0.65_{0.24}^{1.08}$ & hot & $1.05(181)$ & 12.2 & 1376 & see $(5)$ \\
\hline \#18 & Comb. & pn & $1.32_{0.97}^{0.76}$ & $1.07_{0.27}^{0.64}$ & hot & $0.98(82)$ & 11.8 & 1211 & \\
\hline \#23 & Comb. & MOS & $0.71_{0.54}^{1.97}$ & $0.15_{0.11}^{0.18}$ & $1.99^{5.12}$ & $0.98(108)$ & 3.1 & 498 & see $(6)$ \\
\hline \#23 & Comb. & pn & $0.63_{0.25}^{0.54}$ & $0.21_{0.15}^{0.54}$ & $2.05_{1.30}^{5.07}$ & $0.98(48)$ & 2.0 & 821 & \\
\hline \#23 & Comb. & MOS & $0.73_{0.43}^{2.11}$ & $0.14_{0.11}^{0.118}$ & $1.29_{0.99}^{2.78}$ & $0.96(108)$ & 3.0 & 498 & 0.3 solar \\
\hline \#23 & Comb. & $\mathrm{pn}$ & $0.64_{019}^{0.94}$ & $0.20_{0.15}^{0.53}$ & $1.71_{1.21}^{2.20}$ & $0.97(48)$ & 2.1 & 821 & 0.3 solar \\
\hline \#32 & Comb. & MOS & $0.33_{0.18}^{0.58}$ & $0.68_{0.58}^{0.89}$ & $2.83_{241}^{3.51}$ & $1.03(177)$ & 9.8 & 1614 & see (7) \\
\hline \#32 & Comb. & MOS & $0.28_{0.00}^{0.53}$ & $0.75_{0.57}^{0.98}$ & $2.29_{1.93}^{2.68}$ & $0.95(177)$ & 10.4 & 1614 & 0.3 solar \\
\hline
\end{tabular}

Notes. The first part is for objects dominating in one pointing (Sect. 5.1) and the second part (Sect. 5.2) for constant objects for which we combined all pointings (II to VII).

${ }^{(*)}$ Fixed.

(1) The spectral fit with a powerlaw or a single cool mekal already leads to $\chi_{v}^{2}=0.81-0.84$ (d.o.f. $=26$ ). (2) The spectral fit is further supported by the position of source \#30 in the HR diagram (Fig. 4). (3) A wabs *powerlaw gives $N_{\mathrm{H}}=0.21 \times 10^{22} \mathrm{~cm}^{-2}$ and $\Gamma=2.68\left(\chi_{v}^{2}=1.39\right.$, d.o.f. $=21$ ). (4) A simple model of the type wabs $*$ powerlaw gives $N_{\mathrm{H}}=0.19 \times 10^{22} \mathrm{~cm}^{-2}$ and $\Gamma=2.58\left(\chi_{v}^{2}=1.04\right.$, d.o.f. $=106$; in good agreement with its position in Fig. 4). (5) A model of the type wabs*powerlaw yields $N_{\mathrm{H}}=0.19-0.22 \times 10^{22} \mathrm{~cm}^{-2}$ and $\Gamma=1.89-1.79\left(\chi_{v}^{2}(\mathrm{MOS})=1.04\right.$, d.o.f. $=$ $183 ; \chi_{v}^{2}(\mathrm{pn})=1.00$, d.o.f. $\left.=84\right)$ as confirmed by source $\# 18$ position in Fig. 4. (6) Hardness ratios (Fig. 4) and $1-\mathrm{T}$ model fits favour $N_{\mathrm{H}}=0 . \mathrm{cm}^{-2}$ and $k T=0.9-1.5 \mathrm{keV}$. (7) A model of the type wabs *powerlaw suggests an absorbing column of $N_{\mathrm{H}}=0.26 \times 10^{22} \mathrm{~cm}^{-2}$ and a photon index $\Gamma=3.24\left(\chi_{v}^{2}(\mathrm{MOS})=0.99\right.$, d.o.f. $\left.=179\right)$.

some 3.8 times lower than for HD 92607, suggesting a lower $L_{\mathrm{X}} / L_{\mathrm{bol}}$ ratio by a factor of 2 .

\subsection{Source \#13}

Source \#13 is a particularly hard source, suggesting that it is strongly absorbed (see Fig. 4). In Fig. 8, its infrared counterpart is located in the region of reddened objects at $(J-H, H-K)=$ $(1.11,0.53)$. According to these colours it could be a main sequence star with an extinction of about $A_{V} \sim 4.5$. It would then approximately correspond to the colours of an M4V star. However, the magnitude of the optical counterpart $(V=17)$ and the absolute magnitude $M_{V}(\mathrm{M} 4 \mathrm{~V})=11.3$ (Schmidt-Kaler 1982) necessarily constrain the distance modulus to values typical of a nearby object. This proximity is not compatible with the high observed extinction if it is interstellar in origin. On the other hand, source \#13 could also correspond to an A-F star at $A_{V} \sim 8.0$, but here again the distance modulus corresponds to a nearby object incompatible with the huge extinction. Alternatively, an extinction $A_{V} \sim 3.5$ could project this object on the locus of classical T Tauri stars. In this case, the magnitude $K=9.8$ still indicates an apparently bright object. Compared to $V=17$, this suggests that source \#13 is an intrinsically red object which is further obscured. It is remarkable that this source is also variable in the X-ray domain (HS; compatible with a pms status).

\subsection{Source \#14}

Source \#14 is not a hard source, but it is variable. The colours of its infrared counterpart have large errors. It is situated at
$(J-H, H-K)=(0.87,0.51)$ in Fig. 8. It is slightly outside the locus of reddened main sequence objects and could therefore exhibit an intrinsic infrared excess, although this remains within the error bars. An extinction like that of Carina would put the infrared counterpart just on the $\mathrm{T}$ Tauri line. If we admit that source \#14 is at the adopted distance for the Carina region, we derive $M_{V}=4.2$ and $M_{K}=2.5$. In addition, from the count rates of source \#14, we derive $\log L_{X}>30$. This could correspond to an F-G spectral type or to an X-ray-bright pms object.

\subsection{Source \#18}

Source \#18 is among the brightest X-ray sources in the field. The fit of the X-ray spectrum suggests a rather hot object $\left(k T_{1} \sim\right.$ $0.65-1.0 \mathrm{keV}$ and a second hot component, but of undefined temperature). It also points to an absorbing column density of about $10^{22} \mathrm{~cm}^{-2}$. According to Bohlin et al. (1978), this could give $E_{B-V}=1.72$. The magnitudes of the infrared counterpart have large errors and should be used with caution; they indicate $(J-H, H-K)=(0.63,0.43)$. If the object is considered to be at the distance of the Carina region and characterized by the abovementioned extinction, we derive $M_{K}=1.9$ suggesting an FV star (Knödlseder 2000). Such a star would have $M_{V}=2.7-4.0$ and would be at $V=20.2-21.5$. Source \#18 has no counterpart in the GSC2.2; it is situated close to HD 303223, but further south. An eye-inspection of the Digitized Sky Survey clearly reveals an object of magnitude 18-19 (i.e. much brighter than computed above) at the exact position of the X-ray source. A preliminary VLT+FORS1 spectrum of this object indicates a late F-type star with Balmer lines in emission (see Fig. 9). This could 
J.-F. Claeskens et al.: A search for X-ray sources in Carina

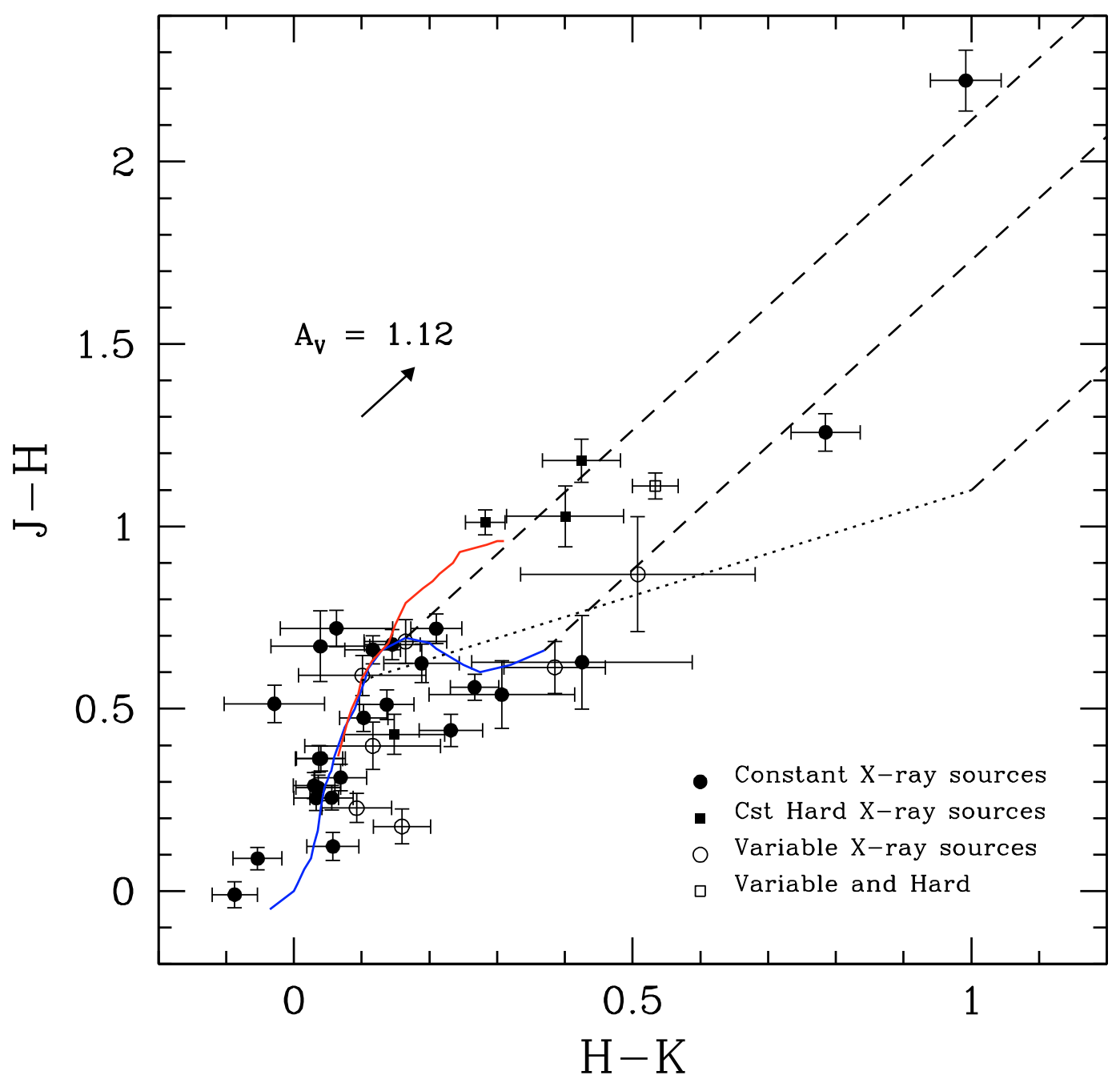

Fig. 8. $J-H$ versus $H-K$ colour-colour diagram. All X-ray sources with an infrared counterpart (with 2MASS photometric colours) are plotted along with their errors (one standard deviation). The colours have been converted to the standard system. The heavy solid lines represent the intrinsic locus of main sequence stars (lower branch) and of giant stars (upper branch). The reddening band for these first stars is delimited by the two upper dashed lines. The dotted line represents the expected locus of dereddened colours for classical T Tauri stars, whereas the lower right dashed line symbolizes the reddening of these pre-main sequence stars. The arrow gives the direction of the interstellar reddening and its amplitude corresponds to the adopted average extinction towards the Carina region.

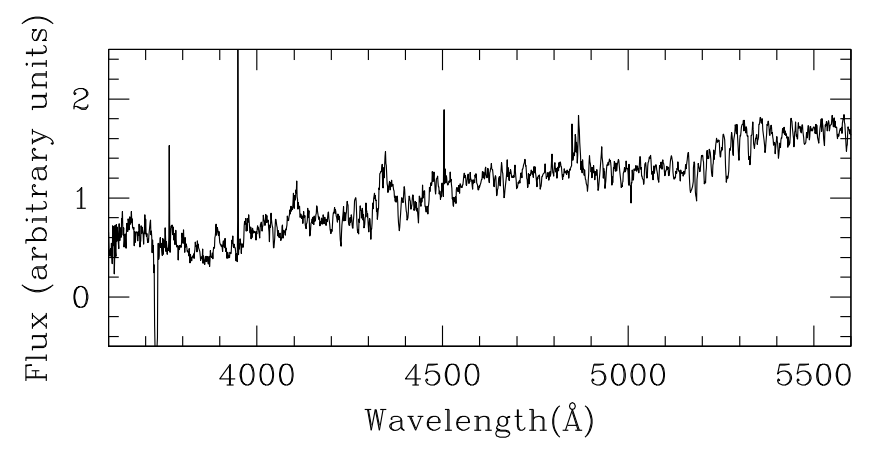

Fig. 9. Optical spectrum of the probable counterpart of source \#18. The spectrum is reminiscent of a late F-star, but Balmer lines are in emission. The emission lines are rather broad.

be an active single or most probably binary star. It could reside in front of the Carina, but it could also be intrinsically brighter than a main sequence star. One of the symbiotics in Crowther \& Smith (1999) has similar infrared colours.

\subsection{Source \#19}

This source is identified with HD 305441. On the basis of the colours of the infrared counterpart, source \#19 is close to the unreddened main sequence. With $(J-H, H-K)=(0.29,0.03)$, it corresponds to a slightly reddened A-F star. The observed $B-V=0.1$ colour further confirms the A nature of the object. This agrees with the A spectral type as reported by Nesterov et al. (1995). Whether the star is a main sequence or a giant, at $V=11.1$, it is a foreground object.

\subsection{Source \#20}

Source \#20 is situated at $(J-H, H-K)=(0.56,0.27)$ and is close to the main sequence branch. It could be an unabsorbed MV star and would then be a foreground object. It could also be a markedly reddened object (roughly $A_{V}=5$.) and would correspond to an obscured $\mathrm{OB}$ object in the Carina region or beyond. Actually, source \#20 is seen in the dark lane. Only a visible spectrum could distinguish between the two possibilities. 
A preliminary spectrum acquired at NTT+EMMI indicates that it is indeed an unknown O-star.

\subsection{Source \#21}

The infrared counterpart of source \#21 is situated at $(J-H, H-K)=(2.22,0.99)$ and is undoubtedly a strongly reddened source. This is further endorsed by the total absence of an optical counterpart. If we adopt $A_{V} \sim 14.2\left(E_{B-V}=4.57\right)$, we deduce $(J-H, H-K, J-K)_{0}=(0.71,0.11,0.80)$. These colours are roughly compatible with a K-MV star or better with a mid-KIII. If it is a main sequence object, it is a foreground object and the extinction is hard to understand. If it is a mid-KIII star, $M_{V}=0.3$ and the object is fainter than $V=20$ (no counterpart visible in Digitized Sky Survey) for distances larger than the one characterized by $D M=5.5$. With $K=12.54$ and $A_{K}=1.59$, the object could be well beyond the Carina. This conclusion is not compatible with the X-ray-derived hardness ratios that only indicate a mildly absorbed spectrum; Fig. 4 suggests an $N_{\mathrm{H}}$ in the range $0.5-1.0 \times 10^{22} \mathrm{~cm}^{-2}$ and thus $E_{B-V}$ in the range $0.9-1.7$. The hardness ratios could however be inaccurate due to the faintness of the source.

\subsection{Source \#23}

The infrared counterpart of source \#23 is very close in Fig. 8 to the non-reddened main sequence locus at $(J-H, H-K)=$ $(0.26,0.03)$. These colours correspond to an F5-F7 star. If we deredden it for the Carina region (as a possible maximum), we obtain the intrinsic colours $(J-H, H-K)_{0}=(0.14,-0.04)$, which rather indicates an F0 spectral type. With observed magnitudes $V=11.4$ and $K=10.2$, this in any case corresponds to an F-star in front of the Carina. This implies a relatively small reddening and thus a low absorption column in front of the X-ray source. This low column density agrees with the position of source \#23 in the HR diagram (Fig. 4) and equivalently with the 1-T model fit, but is opposed to the results of the 2-T model fits reported in Table 5.

\subsection{Source \#30}

The colours of the infrared counterpart of source \#30 are not reliable. The absorbing column deduced from the X-ray spectrum could be compatible with the Carina line-of-sight and distance. From $V=15.8$, we then deduce an absolute magnitude $M_{V}=2.53$ at the Carina location. This roughly corresponds to an FV object. This is however not compatible with the observed $K$ magnitude (see Knödlseder 2000). Nevertheless, we recall that the identity of the visible and the infrared counterparts is doubtful. From $\log L / L_{\odot} \sim 0.5$ (Schmidt-Kaler 1982), we deduce $L_{\text {bol }} \sim 1.2 \times 10^{34} \mathrm{erg} \mathrm{s}^{-1}$. From Table 5, we derive $f_{\mathrm{X}}=$ $14.0 \times 10^{-14} \mathrm{erg} \mathrm{cm}^{-2} \mathrm{~s}^{-1}$, which yields an interstellar corrected flux $f_{\mathrm{X}}^{\mathrm{un}}=16.5 \times 10^{-14} \mathrm{erg} \mathrm{cm}^{-2} \mathrm{~s}^{-1}$. This corresponds to $L_{\mathrm{X}} \sim$ $1.4 \times 10^{32} \mathrm{erg} \mathrm{s}^{-1}$ and to $\log L_{\mathrm{X}} / L_{\mathrm{bol}} \sim-1.9$. This value combined with the possibility that the source is slightly overluminous in $K$, is variable at X-ray wavelengths, and could harbour a hot plasma, all make this object interesting.

\subsection{Source \#36}

Source \#36 corresponds to CD-59 $9^{\circ} 3233$. No spectral type is known for this object, but it must be rather cool. Indeed we have an observed $B-V=1.0$. The infrared colours give
$(J-H, H-K)=(0.48,0.10)$, which corresponds to an unreddened $\mathrm{K} 0-\mathrm{K} 2$ object or to an early GIII. In both cases, this is a foreground object.

\subsection{Source \#37}

The infrared counterpart of source \#37 is at $(J-H, H-K)=$ $(0.23,0.09)$. If we deredden these colours for the Carina, we have $(J-H, H-K)_{0}=(0.11,0.02)$, which roughly gives an A-star (perhaps early F). Starting from $V=14.2$ and $D M+A_{V}=13.27$, we find $M_{V}=0.93$ in good agreement with an A-star in the Carina region. This is an X-ray-variable source, too bright for a typical A-star.

\subsection{Source \#38}

Source \#38 appears at $(J-H, H-K)=(1.26,0.79)$ in Fig. 8. It is the only red object that is markedly outside the reddening band of the main sequence stars. From this point of view, it is an interesting object because it could represent a reddened pms star. Certainly the object has an intrinsic red excess in addition to its extinction. This source has hardness ratios $H R_{1}=-0.04$ and $H R_{2}=0.12$, indicating a mildly hard source, which supports the pms-candidate status. The source is variable, but was rather classified as a long-term variable.

\subsection{Source \#42}

The infrared counterpart of source \#42 has $(J-H, H-K, J-K)=$ $(0.68,0.17,0.85)$. We could associate it with a nearby M0V but the $V-K=3.0$ is already too blue. Therefore, we have to consider a giant and/or a reddened star. If we deredden these colours for the Carina region, we obtain $(J-H, H-K)_{0}=(0.57,0.10)$, which corresponds to a mid-KV or to a G-KIII star. Even at $V=15.1$, the mid-KV should be a foreground object. The GKIII alternative is interesting. For example, if we adopt $A_{V}=2.5$ and the distance of the Carina region, we obtain colours that are reminiscent of a late GIII star. Adopting $M_{V}(\mathrm{GIII})=0.8$, we have $V=15.1$ and thus $D M+A_{V}=14.3$. This is compatible with an object of the Carina region or slightly behind. But then the $K$ magnitude is a little too bright, possibly indicating a weak infrared excess. We note that the absorbing column deduced from the fit is about $A_{V} \sim 3.5$ or more. Owing to the error bars, we should consider that the G-KIII object could possibly belong to the Carina region, but it has a large extinction, part of it possibly intrinsic.

Source \#42 is source \#1 of the catalogue of Antokhin et al. (2008), which concerns the best-studied part of the Carina complex (to the east of the present field). They have five exposures and give the following count rate for the pn: $14.4 \times 10^{-3} \mathrm{cts} / \mathrm{s}$. For MOS1, they have $4.5 \times 10^{-3} \mathrm{cts} / \mathrm{s}$, whereas for MOS2, they have $3.3 \times 10^{-3} \mathrm{cts} / \mathrm{s}$. Compared to our observed count rates (see Table 4), we can conclude that source \#42 is variable because our corrected count rates are higher by a factor of more than 10 . The variability of source \#42 was already noticed on the sole basis of our various pointings.

\section{The diffuse X-ray emission}

As can be seen in Fig. 2, significant diffuse X-ray emission is clearly present in the field, and the definition of the background has been a crucial step for several point sources. When we established the catalogue of X-ray point sources, we detected 


\section{Combined EPIC image}

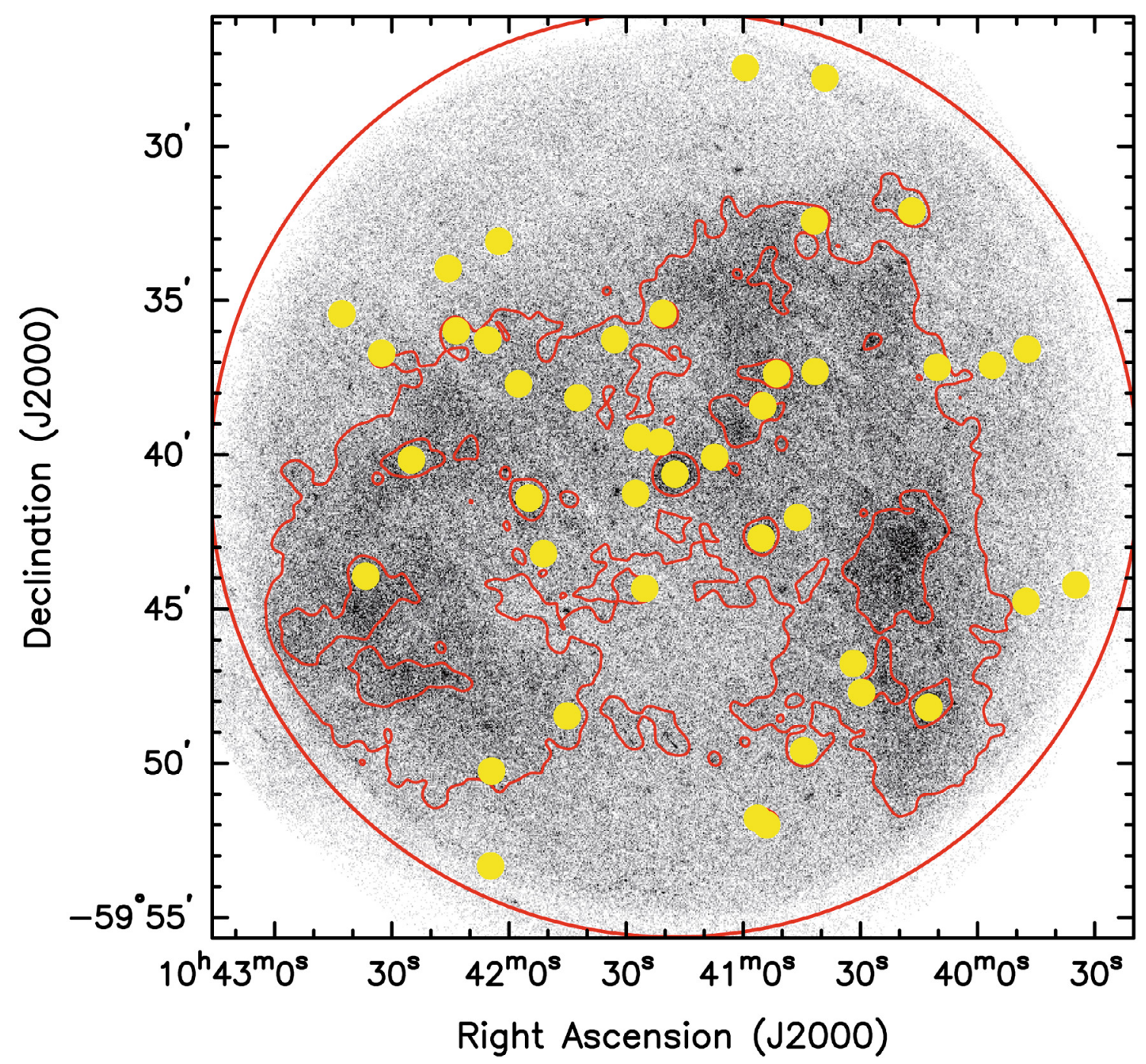

Fig. 10. Diffuse X-ray emission in the Carina region around WR 22 as seen in the combined EPIC image in the total band. The point-like sources were replaced by a yellow/white circle. Contours at 2.0 and $3.2 \mathrm{cts} /$ pixel help in outlining the diffuse emission.

13 extended sources. After an eye inspection, it turned out that all these sources were mere artefacts generated by abrupt changes in the surface brightness of the global diffuse emission, and that none of these 13 sources had a proper existence beyond the one of the global entity. The general diffuse X-ray emission has a shape reminiscent of an asymmetric circumflex accent (see Fig. 10). The diffuse emission is clearly limited to the NE by a straight line going between NW and WNW to between SE and ESE. This borderline corresponds to the edge of the western part of the $\mathrm{V}$-shaped dark lane. This straight line continues to the $\mathrm{SE} / \mathrm{ESE}$ and is still present up to the southern giant molecular cloud (see Brooks et al. 1998) in the neighbourhood of WR 25 in the main Carina region (Antokhin et al. 2008). The border on the west side can also be sketched by a straight line going NNE to SSW. It corresponds to no known structure at other wavelength domains. Finally, towards the south of the field, a corner almost without emission can be spotted.

The diffuse X-ray emission is somewhat patchy and presents several maxima within the field. The variations occur at a typical scale of a few arcmin, i.e. a few pc. The strongest emission is located at a position $\alpha(\mathrm{J} 2000)=10^{\mathrm{h}} 40^{\mathrm{m}} 20^{\mathrm{s}}$, $\delta(\mathrm{J} 2000)=-59^{\circ} 43^{\prime} 22^{\prime \prime}$. We tentatively extracted a spectrum of the diffuse emission in the region of this maximum. The extraction region is characterized by an ellipse oriented NW-SE with semi-axes of $97^{\prime \prime}$ by $60^{\prime \prime}$. The spectrum is well above background up to $1.2 \mathrm{keV}$ but not beyond. It is readily fitted by an absorbed mekal giving a column $N_{\mathrm{H}}=0.21-0.36 \times 10^{22} \mathrm{~cm}^{-2}$ and $k T=0.26-0.31 \mathrm{keV}\left(\chi_{v}^{2}=1.24\right.$, d.o.f. $\left.=43\right)$. It corresponds to an observed flux per unit surface $f_{\mathrm{X}, \mathrm{s}}(0.5-10 \mathrm{keV})=$ $4.8 \times 10^{-14} \mathrm{erg} \mathrm{cm}^{-2} \mathrm{~s}^{-1} \operatorname{arcmin}^{-2}$. A look at Fig. 2 suggests that the temperature is very roughly constant all over the field. The flux corrected for the interstellar extinction is $f_{\mathrm{X}, \mathrm{s}}^{\mathrm{un}}=1.4 \times$ $10^{-13} \mathrm{erg} \mathrm{cm}^{-2} \mathrm{~s}^{-1} \operatorname{arcmin}^{-2}$. The full extracted region (ellipse) is characterized by $L_{\mathrm{X}}=6.3 \times 10^{32} \mathrm{erg} \mathrm{s}^{-1}$. The surface flux value is of the same order of magnitude as the value reported by Seward \& Chlebowski (1982) for the brightest regions of dimension 1.5 (EINSTEIN observations).

The diffuse emission in the remaining part of the present field is two to three times fainter than for the region of the maximum. We thus estimate the observed flux per unit surface to 
be $f_{\mathrm{X}, \mathrm{s}}=1.7 \times 10^{-14} \mathrm{erg} \mathrm{cm}^{-2} \mathrm{~s}^{-1} \operatorname{arcmin}^{-2}$, corresponding to $f_{\mathrm{X}, \mathrm{s}}^{\mathrm{un}}=5.0 \times 10^{-14} \mathrm{erg} \mathrm{cm}^{-2} \mathrm{~s}^{-1} \operatorname{arcmin}^{-2}$. This diffuse emission in the field of view of the pointing covers some $400 \mathrm{arcmin}^{2}$ and the total emission is characterized by $L_{\mathrm{X}}=1.7 \times 10^{34} \mathrm{erg} \mathrm{s}^{-1}$. For the neighbouring field (the main Carina region, see Fig. 1), Seward \& Chlebowski (1982) report a total luminosity of $2 \times$ $10^{35} \mathrm{erg} \mathrm{s}^{-1}$, which can be converted into the unabsorbed surface flux $f_{\mathrm{X}, \mathrm{s}}^{\mathrm{un}}=8.1 \times 10^{-14} \mathrm{erg} \mathrm{cm}^{-2} \mathrm{~s}^{-1} \operatorname{arcmin}^{-2}$. This value is between our two corresponding values, indicating a slightly lower flux in the present global field. The temperature derived by Seward \& Chlebowski is however much higher $(\sim 0.7 \mathrm{keV})$ than that measured in our field, but it could be affected by a lack of spatial resolution. The values we find for the absorbing column density and for the temperature agree very well with those determined by Corcoran et al. (1995) on the basis of the ROSAT data. These authors report a surface flux (ROSAT band) of $f_{\mathrm{X}, \mathrm{s}}=1.0 \times 10^{-13} \mathrm{erg} \mathrm{cm} \mathrm{cm}^{-2} \mathrm{~s}^{-1} \operatorname{arcmin}^{-2}$ (region 6, south of $\eta$ Car), $f_{\mathrm{X}, \mathrm{s}}=1.4 \times 10^{-13} \mathrm{erg} \mathrm{cm}^{-2} \mathrm{~s}^{-1} \operatorname{arcmin}^{-2}$ (region 1, south of WR 25) and $f_{\mathrm{X}, \mathrm{s}}=3.11 \times 10^{-14} \mathrm{erg} \mathrm{cm}^{-2} \mathrm{~s}^{-1} \mathrm{arcmin}^{-2}$ (region 2, north-west of WR 25). They also introduce a possible second optically thin thermal plasma for region 1 at a temperature of $0.7 \mathrm{keV}$. Recently, Hamaguchi et al. (2007) reobserved the $\eta$ Car field with Suzaku and, thanks to better quality data, proposed 3-T models with $N_{\mathrm{H}}=0.18-0.24 \times 10^{22} \mathrm{~cm}^{-2}$, $k T_{1}=0.13-0.24 \mathrm{keV}, k T_{2}=0.56-0.60 \mathrm{keV}$, and a hotter component. The corresponding surface fluxes are $f_{\mathrm{X}, \mathrm{s}}(0.5-2.0 \mathrm{keV})=$ $7.0-9.5 \times 10^{-14} \mathrm{erg} \mathrm{cm}^{-2} \mathrm{~s}^{-1} \operatorname{arcmin}^{-2}$ and $f_{\mathrm{X}, \mathrm{s}}(2.0-8.0 \mathrm{keV})=$ $3.5-3.8 \times 10^{-14} \mathrm{erg} \mathrm{cm}^{-2} \mathrm{~s}^{-1} \operatorname{arcmin}^{-2}$; the two figures correspond to their north and south fields respectively. Our results for the WR 22 field fit well with the dominating cooler $k T_{1}=$ $0.2-0.3 \mathrm{keV}$ temperature in the North reported by other authors for the main Carina field. However, the flux per unit surface seems lower in the field we studied here.

One of the interpretations of the origin of the diffuse emission as seen by EINSTEIN was the presence of numerous unresolved pms objects. Recently, Evans et al. (2003), analysing Chandra data, concluded that at least half of the emission is actually diffuse. The situation is even more extreme in this WR 22 field that exhibits no strong sign of a large population of pms objects. The hot diffuse medium could be due to wind shocks owing to early-type stars and/or possibly to supernova remnants. Townsley et al. (2003) concluded that the relative contributions of these two origins are still unknown. The more recent studies of Hamaguchi et al. (2007) and of Ezoe et al. (2009) concerning the main Carina region and another region further east, revealed chemical abundance effects that could help to solve the question of the origin. This study is however not possible here due to the limited number of counts available.

\section{Conclusions}

We performed an X-ray survey in a small field situated in the direction of the Carina region. The surveyed area is located slightly to the west of the well-known region containing the clusters $\operatorname{Tr} 14$ and $\operatorname{Tr} 16$, and $\eta$ Car, and is centred on the massive WR+O binary WR 22. This field is poorly studied and no deep photometric work covers it. We established a catalogue of 43 bona-fide X-ray sources, most of which were unknown before the XMM-Newton observations. We performed the first steps towards the identification of the sources using various optical/infrared catalogues.

We identified one interesting active source exhibiting a late F-spectrum, but also with Balmer lines in emission. Several of the X-ray sources clearly belong to the Carina complex. One of the sources is associated with an obscured previously unknown $\mathrm{O}$ star, indicating that we are detecting objects beyond the near side of the Carina region. No clustering of the sources seems to be present in the field, which implies the absence of outstanding open clusters like Tr 14 and Tr 16 (see Antokhin et al. 2008). Though we are away from the open cluster centres, a few X-ray sources have an obscured counterpart and/or a counterpart exhibiting slight infrared excesses and are thus good pms candidates that perhaps sample the X-ray-bright end of the population. We also showed that the present field exhibits a truly diffuse $\mathrm{X}$-ray emission.

We plan to continue the identification process and to perform deep photometry and spectroscopy in the field. It is particularly interesting because the line of sight is tangential to a spiral feature, while it avoids the well-known populated open clusters of the main part of the Carina complex.

Acknowledgements. The authors are greatly indebted to the Belgian FNRS for multiple support. They also acknowledge financial support through PRODEX for the XMM and INTEGRAL projects. Further support from the Belgian Federal Science Policy Office through contracts "Pôle d'Attraction Interuniversitaire" $\mathrm{P} 4 / 05$ and P5/36 and from the Communauté Française de Belgique through Action de Recherche Concertée (Académie Wallonie-Europe), is also duly acknowledged. We further thank A. Detal, our system manager, for various help to users, in particular concerning the SAS. This research has made use of the SIMBAD database, operated at CDS, Strasbourg, France.

\section{References}

Albacete-Colombo, J. F., Méndez, M., \& Morrell, N. 2003, MNRAS, 346, 704 Albacete-Colombo, J. F., Damiani, F., Micela, G., Sciortino, S., \& Harnden, F. R. Jr. 2008, A\&A, 490, 1055

Anders, E., \& Grevesse, N. 1989, Geochim. Cosmochim. Acta, 53, 197

Antokhin, I. I., Rauw, G., Vreux, J.-M., van der Hucht, K. A., \& Brown, J. C. 2008, A\&A, 477, 593

Arnaud, K. A. 1996, in Astronomical Data Analysis Software and Systems V, ed. G. H. Jacoby, \& J. Barnes, ASP Conf. Ser., 101, 17

Arnaud, K. A. 1999, in HEAD meeting \#4, BAAS, 31, 734

Berghöfer, T. W., Schmitt, J. H. M. M., \& Cassinelli, J. P. 1996, A\&AS, 118, 481 (erratum: A\&AS, 121, 212)

Berghöfer, T. W., Schmitt, J. H. M. M., Danner, R., \& Cassinelli, J. P. 1997, A\&A, 322, 167

Bertin, E., \& Arnouts, S. 1996, A\&AS, 117, 393

Bessell, M. S., \& Brett, J. M. 1988, PASP, 100, 1134

Bohlin, R. C., Savage, B. D., \& Drake, J. F. 1978, ApJ, 224, 132

Brooks, K. J., Whiteoak, J. B., \& Storey, J. W. V. 1998, PASA, 15, 202

Brooks, K. J., Storey, J. W. V., \& Whiteoak, J. B. 2001, MNRAS, 327, 46

Carpenter, J. M. 2001, AJ, 121, 2851

Carraro, G., Romaniello, M., Ventura, P., \& Patat, F. 2004, A\&A, 418, 525

Chlebowski, T., Harnden, F. R. Jr., \& Sciortino, S. 1989, ApJ, 341, 427

Churchwell, E. 2000, in Massive Star Birth, IAU General Assembly, 24, August, Manchester, England, Joint Discussion, 3, 8

Corcoran, M. F., Swank, J., Rawley, G., et al. 1995, Rev. Mexicana Astron. Astrofis. Ser. Conf., 2, 97

Cox, P. 1995, Rev. Mexicana Astron. Astrofis. Ser. Conf., 2, 105

Crowther, P. A., \& Smith, L. J. 1999, MNRAS, 308, 82

Cruddace, R. G., Hasinger, G. R., \& Schmitt, J. H. 1988, in Astronomy from large databases: Scientific objectives and methodological approaches, ed. F. Murtagh, \& A. Heck, European Southern Observatory, Garching bei München, Germany, ESOC, 28, 177

Cutri, R. M., Skrutskie, M. F., van Dyk, S., et al. 2003, 2MASS All Sky Catalog of point sources, NASA/IPAC Infrared Science Archive

Damiani, F., Flaccomio, E., Micela, G., et al. 2004, ApJ, 608, 781

DeGioia-Eastwood, K., Throop, H., Walker, G., \& Cudworth, K. M. 2001, ApJ, 549,578

Dickel, H. R. 1974, A\&A, 31, 11

Dufour, R. J., van Orsow, D., Walter, D. K., et al. 1998, Rev. Mexicana Astron. Astrofis. Ser. Conf., 7, 217

Evans, N. R., Seward, F. D., Krauss, M. I., et al. 2003, ApJ, 589, 509

Evans, N. R., Schlegel, E. M., Waldron, W. L., et al. 2004, ApJ, 612, 1065

Ezoe, Y., Hamaguchi, K., Gruendl, R. A., et al. 2009, PASJ, 61, S123

Feigelson, E. D., \& Montmerle, T. 1999, ARA\&A, 37, 363 
Feigelson, E. D., Townsley, L., Güdel, M., \& Stassun, K. 2007, in Protostars and Planets V, ed. B. Reipurth, D. Jewitt, \& K. Keil (Tucson: University of Arizona Press), 313

Forte, J. C. 1976, A\&AS, 25, 271

Gagné, M., Skinner, S. L., \& Daniel, K. J. 2004, ApJ, 613, 393

Garmire, G., Feigelson, E. D., Broos, P., et al. 2000, AJ, 120, 1426

Giacconi, R., Rosati, P., Tozzi, P., et al. 2001, ApJ, 551, 624

Gosset, E., Nazé, Y., Sana, H., Rauw, G., \& Vreux, J.-M. 2009, A\&A, 508, 805

Grabelsky, D. A., Cohen, R. S., Bronfman, L., \& Thaddeus, P. 1988, ApJ, 331, 181

Güdel, M., \& Nazé, Y. 2009, A\&A Rev., 17, 309

Hamaguchi, K., Petre, R., Matsumoto, H., et al. 2007, PASJ, 59, S151

Hofner, P., \& Churchwell, E. 1997, ApJ, 486, L39

Hofner, P., Delgado, H., Whitney, B., Churchwell, E., \& Linz, H. 2002, ApJ, 579,95

Houk, N., \& Cowley, A. P. 1975, Catalogue of two dimensional spectral types for the HD stars, Vol. 1, Michigan Spectral Survey, Ann Arbor, Dep. Astron., Univ. Michigan, 1

Howarth, I. D., \& Prinja, R. K. 1989, ApJS, 69, 527

Jansen, F., Lumb, D., Altieri, B., et al. 2001, A\&A, 365, L1

Jeffries, R. D., Thurston, M. R., \& Pye, J. P. 1997, MNRAS, 287, 350

Kaastra, J. S. 1992, An X-ray spectral code for optically thin plasmas, Internal SRON-Leiden Report

Kamata, Y., Koyama, K., Tsuboi, Y., \& Yamauchi, S. 1997, PASJ, 49, 461

Knödlseder, J. 2000, A\&A, 360, 539

Megeath, S. T., Cox, P., Bronfman, L., \& Roelfsema, P. R. 1996, A\&A, 305, 296

Mewe, R., Gronenschild, E. H. B. M., \& van den Oord, G. H. J. 1985, A\&AS, 62, 197

Meyer, M. R., Calvet, N., \& Hillenbrand, L. A. 1997, AJ, 114, 288

Motch, C., Herent, O., \& Guillout, P. 2003, Astron. Nachr., 324, 61

Nesterov, V. V., Kuzmin, A. V., Ashimbaeva, N. T., et al. 1995, A\&AS, 110, 367

Neuhäuser, R. 1997, Science, 276, 1363

Pires, A. M., Motch, C., Turolla, R., Treves, A., \& Popov, S. B. 2009, A\&A, 498, 233
Preibisch, T., \& Zinnecker, H. 2002, AJ, 123, 1613

Rathborne, J. M., Burton, M. G., Brooks, K. J., et al. 2002, MNRAS, 331, 85

Rauw, G., Nazé, Y., Gosset, E., et al. 2002, A\&A, 395, 499

Rieke, G. H., \& Lebofsky, M. J. 1985, ApJ, 288, 618

Ryter, C. E. 1996, Ap\&SS, 236, 285

Sana, H., Stevens, I. R., Gosset, E., Rauw, G., \& Vreux, J.-M. 2004, MNRAS, 350,809

Sana, H., Gosset, E., Rauw, G., Sung, H., \& Vreux, J.-M. 2006a, A\&A, 454, 1047

Sana, H., Rauw, G., Nazé, Y., Gosset, E., \& Vreux, J.-M. 2006b, MNRAS, 372, 661

Sana, H., Rauw, G., Sung, H., Gosset, E., \& Vreux, J.-M. 2007, MNRAS, 377, 945

Sanchawala, K., Chen, W. P., Lee, H. T., et al. 2007, ApJ, 656, 462

Schlegel, D. J., Finkbeiner, D. P., \& Davis, M. 1998, ApJ, 500, 525

Schmidt-Kaler, T. 1982, Physical Parameters of the Stars, ed. K. Schaifers, \& H. H. Voigt, Vol. 2b of Landolt-Börnstein, Numerical Data and Functional Relationships in Science and Technology, New Series, Group IV (Berlin: Springer-Verlag), 14

Seward, F. D., \& Chlebowski, T. 1982, ApJ, 256, 530

Seward, F. D., Forman, W. R., Giacconi, R., et al. 1979, ApJ, 234, L55

Smith, N. 2006, MNRAS, 367, 763 (Erratum: 368, 1983)

Smith, N., Egan, M. P., Carey, S., et al. 2000, ApJ, 532, L145

Smith, N., Bally, J., \& Morse, J. A. 2003, ApJ, 587, L105

Stevens, I. R., Blondin, J. M., \& Pollock, A. M. T. 1992, ApJ, 386, 265

Strüder, L., Briel, U., Dennerl, K., et al. 2001, A\&A, 365, L18

Tapia, M., Roth, M., Vázquez, R. A., \& Feinstein, A. 2003, MNRAS, 339, 44

Townsley, L. K., Feigelson, E. D., Montmerle, T., et al. 2003, ApJ, 593, 874

Turner, M. J. L., Abbey, A., Arnaud, M., et al. 2001, A\&A, 365, L27

Vuong, M. H., Montmerle, T., Grosso, N., et al. 2003, A\&A, 408, 581

Walter, F. M. 1992, AJ, 104, 758

Watson, M. G., Schröder, A. C., Fyfe, D., et al. 2009, A\&A, 493, 339 